\title{
On crop vector-borne diseases. Impact of virus lifespan and contact rate on the traveling-wave speed of infective fronts
}

\author{
M. Chapwanya ${ }^{+}$, and Y. Dumont ${ }^{\dagger *}$ \\ + Department of Mathematics \& Applied Mathematics, University of Pretoria, \\ Pretoria 0002, South Africa, \\ $\dagger$ CIRAD, Umr AMAP, Montpellier, France
}

December 18, 2017

\begin{abstract}
Diseases, in particular, vector-borne diseases are very important issues in crop protection. However, despite their impact on food safety, very few mathematical models have been developed in order to improve on control strategies. Motivated by existing literature, we begin by considering a temporal model of vector-borne diseases in (annual) crops. Using appropriate mathematical methods, we show the existence of threshold parameters and discuss several control strategies based on the model. Then, the model is modified to include spatial component in order to take into account that vectors are moving. We study both theoretically and numerically the related system and other subsystems that are easier to handle using the theory of monotone systems. We show that traveling wave solutions may exist, with traveling wave speed dependent on the virus lifespan and the contact rate between the pest and the crop. Finally, we discuss the consequences in terms of control strategies.
\end{abstract}

Keywords: Vector-borne disease; barrier crop; partially degenerate reaction diffusion system; cooperative system; traveling wave speed; numerical simulations.

In the next decade, with the increase in world population and the reduction of lands dedicated to crops, compounded with climate change, there is a challenge to sustain and/or increase food production. Even now in many places around the world, food security is an important issue. It is well known that one quarter of the total crop production is lost because of pests and diseases. Despite many programs and studies to improve on food security, these losses have increased mainly because of the emergence of new diseases or new pests and also because of increasing climatic events. This work focuses on understanding vector-borne diseases in plants with the aim of improving on control strategies.

Like humans and animals, plants have to deal with viruses and/or obligate parasites. However, plant viruses have difficulties to overcome and there are some transmission restrictions due to plant immobility. Contrary to humans or animals, there is no plant-to-plant contact, at least in standard crops, and the wall made of cellulose and pectin that surround all plant cells limit the entries and exits of viruses. To circumvent these obstacles, plant viruses have developed different strategies to transfer efficiently from one host to another. In this case, plant viruses need some outside partners or some transport devices that allow an efficient transmission to new hosts. Such devices are usually called vectors, and are found among parasiti fungi, root nematodes and plant-feeding arthropods, particularly insects.

To be efficient in virus transmission, these vectors are able to break the cellulose and pectin barriers using their feeding organs, usually the stylet, and then move frequently from plant to plant. Thus any organism that may feed on an infected plant and travel between plants can potentially transport the virus and thus transmit it to healthy plants. In some sense, plant vectors are involved in the same way mosquitoes are involved in the transmission of diseases such as Malaria [34], Dengue or Chikungunya [11, 12]. However, in the plant kingdom, transmission mechanisms are more complex.

\footnotetext{
${ }^{*}$ Corresponding author: yves.dumont@cirad.fr
} 
Indeed, a very important difference between animal viruses and plant viruses lie in the transmission process. For animal/human viruses, no mechanical or direct transmission is possible. For example, an extrinsic incubation period is necessary such that an infected mosquito becomes infective, i.e., to be able to infect susceptible humans. For plant, virus transmission characteristics are different and depend on the interaction between the virus and the vector [19]. Mechanical and biological transmissions are considered to be the main way for viruses transmission by arthropod vectors. However theses terms are not always clear and do not represent efficiently the mechanism of insect transmission of plant-infecting viruses.

Following [19], it is necessary to consider different types of transmission mechanisms described by time events. Three main transmission modes have been defined:

- the non-persistent mode, with viruses acquired within seconds, and not retained for more than a few hours by their vectors.

- the semi-persistent mode, with viruses acquired within minutes and retained for several hours.

- the persistent mode if they remain in the vector for the rest of its life. In that case the acquisition and inoculation times, as well as the latent periods are of days.

An additional classification has been considered by biologists. They distinguish viruses that remain outside the vectors, from those traversing the intestine via the body lumen to the salivary glands. The first type of viruses enter the category of non circulative viruses that have a more superficial and transient relationship with the vector. Transmissible virus particles attach only to the exterior mouthpieces of the insect from which they are released into a new host. In the animal kingdom, this kind of transmission is also called mechanical transmission and is important in the epidemiology of many animal diseases. In particular, many Diptera species are responsible for mechanical transmission. The second type of plant viruses are called circulative viruses and they may be inoculated with the saliva into a new host plant. A circulative virus can further be classified as circulative non propagative or circulative propagative, if, in addition, the virus replicates within the vector. In general, non circulative viruses are non-persistent or semi-persistent, while circulative viruses are semi-persistent or persistent.

Altogether plant viruses strongly depend on vectors for their transmission and survival. As a result modeling has to take into account the vector population and the type of virus. Moreover, it seems natural to consider vector control as an efficient method to reduce the epidemiological risk.

Aphids and whiteflies are known to transmit more than 500 non-persistent, semi-persistent or persistent viruses, $[7,31,14]$. Among them, around 300 are non-persistent viruses. That is why, in particular, aphidborne non-persistent diseases are the most damaging around the world. In addition to that, most of the impacted crops or plants are not suitable for the reproduction or even the survival of aphids.

Indeed, once landed on a plant, aphids first probe the prospective food source by short, only seconds lasting intracellular punctures in epidermis and mesophyll cells that do not even kill the punctured cells. After these exploratory punctures and they judge the plant as suited, the aphids insert their proboscis-like mouthpieces (stylets) into the phloem and feed from its sap for time spans that may exceed several hours. Depending on the tissues they infect, plant viruses can be acquired by aphids during either or only one of the two puncture phases. For example, Luteoviruses are only acquired from the vascular tissues, whereas Cauliflower mosaic virus (CaMV) is acquired from both vascular and no-vascular tissues [32]. CaMV is one of the most studied non-persistent virus. Here, we list some examples of non-persistent plant viruses for which aphids are the principal vectors:

- the Potato virus Y causes the most important aphid-borne virus diseases in potato crops.

- the Cucumber mosaic virus (CMV) was first found on cucumber, but it can infect a wide varieties of plants, including vegetables. It can be transmitted by aphids (between 60 and 80 species), but also by humans and seeds.

- Tomato chlorosis virus (ToCV) is a non-circulative-transmitted crinivirus which can be transmitted by whiteflies such as Bemisia tabacci, [15].

According to [15], plants infected by viruses like CMV or ToCV produce volatiles that attract aphids or whiteflies. This is called the Host Manipulation Hypothesis [6, 22]. However, for circulative virus, we may 
also have the Vector Manipulation Hypothesis [6, 22]. This is a strategy by plant pathogens to enhance their spread to new hosts through their effects on mobile vectors, inducing, for instance, a migratory behavior [6]. Thus, virus transmission can be rather complex. That is why a good knowledge on Host-Vector-Virus interactions is necessary in order to develop appropriate vector control or crop protection strategies. It is important to note that non-persistent viruses are related to annual crops.

For decades, mathematical epidemiology has mainly focused on human diseases [1] and vector borne diseases, like Malaria [34, 28], Dengue or Chikungunya [11, 12], using various mathematical theories [1], with some great successes or achievements. One of the greatest achievement is the so-called Mosquito Theorem, proved by Sir Ronald Ross [34], who, using a mathematical model, was able to show that reducing the anopheles population is necessary (but not sufficient!) to lower the epidemiological risk. This theorem is more or less still used today for many vector-borne diseases. Another great achievement is the famous basic reproduction number $\mathcal{R}_{0}$, a threshold parameter related to some parameters of the model and aggregating important informations related to the dynamics of the disease, [1,9]. In general, when $\mathcal{R}_{0}<1$, the disease is supposed to die out, while the disease becomes endemic when $\mathcal{R}_{0}>1$.

It is clear that for human and animal diseases, mathematical models have been helpful to better understand complex systems and improve health control strategies. However, from a modeling point of view, compared to human vector-borne diseases, plant or crop vector-borne diseases have been poorly studied though they use a similar compartmental approach leading to almost the same kind of equations. There are, however, some additional changes depending on the type of virus, vectors and the relationship between the virus and the vector.

The aim of this study is to extend the model studied in [3] by taking into account different control strategies (like sanitary harvest and/or the use of Barrier plants), and vector displacement. In section 1, we recall the temporal model, consider and study some control strategies like sanitary harvest or barrier plants. Then, in section 2, we build an ODE-PDE Model in order to take into account that vectors can move while plants are stationary. In section 3, we consider a simplified version to show the existence of traveling wave solutions, with a speed dependent on the virus lifespan or the daily contact rate between vectors and plants. Finally, in section 4, we present simulations to illustrate the theoretical results. The paper ends with a discussion and further possible ways of investigations both mathematically and experimentally.

\section{The temporal Vector-borne plant disease model}

We begin with the model developed in [3], that is summarized in the following compartmental diagram:

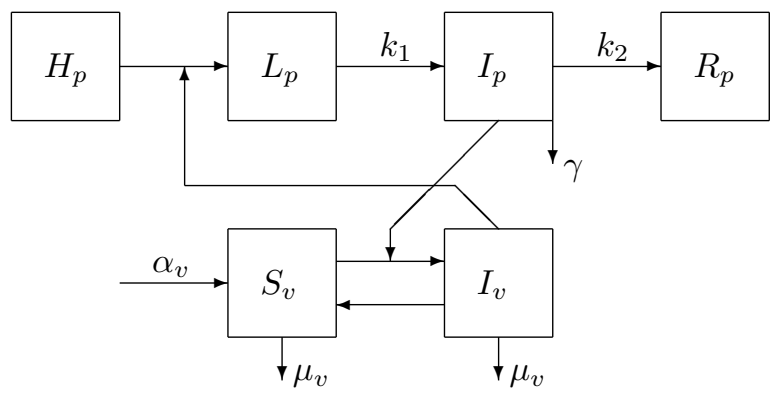

Figure 1: The Crop Vector-borne disease compartmental diagram

The plant population is divided into four epidemiological states: $H_{p}$, the healthy plants, $L_{p}$, the infected but not infectious plants, $I_{p}$, the infected plants, and $R_{p}$, the recovered plants. The insect population is divided into two epidemiological states: $S_{v}$, the susceptible vectors and $I_{v}$, the infectious vectors. We assume that in the field or the greenhouse, the total plant plant population is given by $H_{p}+L_{p}+I_{p}+R_{p}=K$ for all time $t>0$. We further assume here that the virus can impact the life-span of the plant. Here $\frac{1}{k_{1}}$ is the 
mean time a plant stays in the compartment $L_{p}$ and $\frac{1}{k_{2}+\gamma}$ represents the mean time a plant is infective, i.e., stay in the infective compartments.

The vector population is assumed to follow the logistic growth equation

$$
\frac{d V}{d t}=\left(\alpha_{v}-\left(\mu_{1}+\mu_{2} V\right)\right) V
$$

where $\alpha_{v}-\mu_{1}>0$ is the net growth rate of the vector population and $\mu_{2}$ is the death-rate due to the density effect. We set $\mathcal{N}=\frac{\alpha_{v}}{\mu_{1}}$, that is the basic offspring number related to the vector population. We assume that $\mathcal{N}>1$ for the rest the paper.

We consider a mass action principle to model the disease transmission: $\phi$, the contact rate, represents the mean number of plants visited by one insect per unit of time (day), $b$ is the probability of being inoculated during a visit by an infected vectors $I_{v}$. Thus $\phi b I_{v}$ represents the amount of plants infected by all infectious vectors per unit of time. Similarly $\phi a I_{p}$ represents the amount of vectors infected by $I_{p}$ infectious plants per unit of time, where $a$ is the probability of being inoculated during a visit on an infected plant.

At this stage, we recover a model similar to some standard (human) vector-borne disease models. However, we have to take into account that plant virus might be non- or semi-persistent (non-circulative), meaning that the vector does not stay infective its life long (and the virus does not replicate) but becomes susceptible again. The recovery rate depends on the lifespan of the virus, say $1 / \delta$, that is not constant. This may depend on the number of infective plants, $I_{p}$. In other words, the larger $I_{p}$ the longer the vectors will stay in the infective compartments. We model this factor by $\exp \left(-\frac{\phi a I_{p}}{\delta K}\right)$. Of course, when $\delta$ is large, this factor is close to one, and can be neglected. On the other hand, when $\delta=0$, meaning that once the vector is infected it remains infected until its death, the factor is assumed to be zero.

Contrary to [3], we consider that (severely) infective plants can be harvested daily, in other words the parameter $\gamma$ represents the sanitary daily harvest of infective plants. Harvesting infective plants is a way to reduce the spreading of the epidemic. Of course, this is only possible if the symptoms of the disease are visible.

Finally, we obtain the following system of ordinary differential equations (ODEs):

$$
\left\{\begin{array}{l}
\frac{d H_{p}}{d t}=-\phi b I_{v} \frac{H_{p}}{K} \\
\frac{d L_{p}}{d t}=\phi b I_{v} \frac{H_{p}}{K}-k_{1} L_{p} \\
\frac{d I_{p}}{d t}=k_{1} L_{p}-\left(k_{2}+\gamma\right) I_{p} \\
\frac{d R_{p}}{d t}=k_{2} I_{p}
\end{array}\right.
$$

and

$$
\left\{\begin{array}{l}
\frac{d S_{v}}{d t}=\alpha_{v} V-\left(\mu_{1}+\mu_{2} V\right) S_{v}-\phi a S_{v} \frac{I_{p}}{K}+\delta I_{v} e^{-\frac{\phi a I_{p}}{\delta K}} \\
\frac{d I_{v}}{d t}=\phi a S_{v} \frac{I_{p}}{K}-\delta I_{v} e^{-\frac{\phi a I_{p}}{\delta K}}-\left(\mu_{1}+\mu_{2} V\right) I_{v} .
\end{array}\right.
$$

It is important to notice that the total plant population verifies $\frac{d K}{d t}=-\gamma I_{p}$, such that when $\gamma>0$ there is some loss in the plant population. The rest of the study of system (1) and (2) follows more or less [3], except the computation of the basic reproduction number that was wrongly estimated in [3]. Using a suitable change of variables, i.e., $h_{p}=\frac{H_{p}}{K}, l_{p}=\frac{L_{p}}{K}, i_{p}=\frac{I_{p}}{K}, r_{p}=\frac{R_{p}}{K}, s_{v}=\frac{S_{v}}{V}, i_{v}=\frac{I_{v}}{V}$, it suffices to study the simplified system: 


$$
\left\{\begin{aligned}
\frac{d h_{p}}{d t} & =\gamma h_{p} i_{p}-\phi b i_{v} h_{p} \rho \\
\frac{d l_{p}}{d t} & =\gamma l_{p} i_{p}+\phi b i_{v} h_{p} \rho-k_{1} l_{p} \\
\frac{d i_{p}}{d t} & =\gamma i_{p}^{2}+k_{1} l_{p}-\left(k_{2}+\gamma\right) i_{p} \\
\frac{d i_{v}}{d t} & =\phi a\left(1-i_{v}\right) i_{p}-\delta i_{v} e^{-\frac{\phi a i_{p}}{\delta}}-\alpha_{v} i_{v}
\end{aligned}\right.
$$

where $\rho=V / K$ and

$$
\left\{\begin{array}{l}
\frac{d K}{d t}=-\gamma i_{p} K \\
\frac{d V}{d t}=\mu_{2}\left(\frac{\alpha_{v}-\mu_{1}}{\mu_{2}}-V\right) V
\end{array}\right.
$$

with $K(0)=K_{0}>0$, and $V(0)=V_{0} \geq 0$. We set $\tilde{V}=\frac{\alpha_{v}-\mu_{1}}{\mu_{2}}$ and $\tilde{\rho}=\frac{\tilde{V}}{K_{\infty}}$, where $K_{\infty}=\lim _{t \rightarrow \infty} K(t)>0$. It is straightforward to show that $\rho$ is always non negative since $\left.\left.(K, V) \in \Omega_{K, V}=\right] 0, K_{0}\right] \times\left[0, \frac{\alpha_{v}-\mu_{1}}{\mu_{2}}\right]$. Then, setting

$$
\Omega_{p}=\left\{x=\left(h_{p}, l_{p}, i_{p}, i_{v}\right)^{T} \in \mathbb{R}_{+}^{4} \mid \begin{array}{c}
h_{p}+l_{p}+i_{p} \leq 1 \\
i_{v} \leq 1
\end{array}\right\},
$$

system (3)-(4) is mathematically and biologically well posed in $\Omega=\Omega_{p} \times \Omega_{K, V}$ leading to the following

Theorem 1. Assuming that all initial conditions lie in $\Omega$, then (3)-(4) has a unique solution that remains in $\Omega$ for all positive time $t$.

Proof. Obviously, the right-hand side of system (3)-(4) is a continuously differentiable map $\left(C^{1}\right)$. Then, by the Cauchy-Lipschitz theorem, system (3)-(4) provides a unique maximal solution. It remains to show that $\Omega$ is forward-invariant. First, let us note that system (3) can be rewritten as $\frac{d x}{d x}=A(x, V, K) x$, with $A(x)$ being a Metzler Matrix (all off diagonal terms are nonnegative) for $x \in \mathbb{R}_{+}^{4}$, and $(K, V) \in \Omega_{K, V}$. Thus, $\mathbb{R}_{+}^{4}$ is invariant by system (3), meaning that if $x(0) \geq 0$, then $x(t) \geq 0$, for all time $t>0$. It is easy to verify that if $h_{p}+l_{p}+i_{p} \leq 1$ then $\frac{d h_{p}}{d t}+\frac{d l_{p}}{d t}+\frac{d i_{p}}{d t}=\gamma\left(h_{p}+l_{p}+i_{p}-1\right) i_{p}-k_{2} i_{p} \leq-k_{2} i_{p} \leq 0$; if $i_{v} \leq 1$, then $\frac{d i_{v}}{d t} \leq-\alpha_{v} i_{v} \leq 0$. Therefore, we deduce that none of the orbits can leave $\Omega$.

Since we already know the equilibria of system $(4)$, i.e $\left(K_{\infty}, \tilde{V}\right)$, we focus on equilibria of system (3) that belongs to the following set

$$
\mathcal{P}=\left\{x=\left(h_{p}, 0,0,0\right)^{T}: 0 \leq h_{p} \leq 1\right\} \subset \Omega_{p} .
$$

The stability of equilibria is studied using the basic reproduction ratio which is typically derived using the next generation matrix $(N G M)$ approach. For every $x \in \mathcal{P}$ we compute $N G M\left(h_{p}\right)$ [39], and derive the reproduction ratio at $h_{p}$. We rewrite our system for the vector $y=\left(l_{p}, i_{p}, i_{v}\right)^{T}$ in the form

$$
\begin{aligned}
\frac{d y}{d t} & =\mathcal{F}(x)-\mathcal{V}(x) \\
& =\left(\begin{array}{c}
\phi b i_{v} h_{p} \rho \\
0 \\
\phi a i_{p}
\end{array}\right)-\left(\begin{array}{c}
k_{1} l_{p} \\
\left(k_{2}+\gamma\right) i_{p}-k_{1} l_{p} \\
\phi a i_{v} i_{p}+\delta i_{v} e^{-\frac{\phi a_{p}}{\delta}}+\alpha_{v} i_{v}
\end{array}\right) .
\end{aligned}
$$


Then, computing the Jacobian $F(V)$ for at equilibrium, we have

$$
\begin{aligned}
N G M(x) & =F V^{-1} \\
& =\left(\begin{array}{ccc}
0 & 0 & \phi b \rho h_{p} \\
0 & 0 & 0 \\
0 & \phi a & 0
\end{array}\right)\left(\begin{array}{ccc}
k_{1} & 0 & 0 \\
-k_{1} & k_{2}+\gamma & 0 \\
0 & 0 & \delta+\alpha_{v}
\end{array}\right)^{-1} \\
& =\left(\begin{array}{ccc}
0 & 0 & \frac{\phi b \rho h_{p}}{\delta+\alpha_{v}} \\
0 & 0 & 0 \\
\frac{\phi a}{k_{2}+\gamma} & \frac{\phi a}{k_{2}+\gamma} & 0
\end{array}\right) .
\end{aligned}
$$

Then, according to [39], the reproduction ratio at $x$ is defined as the spectral radius of $N G M(x)$. Hence we have

$$
\mathcal{R}^{2}(x)=\frac{\phi^{2} a b}{\left(k_{2}+\gamma\right)\left(\alpha_{v}+\delta\right)} \tilde{\rho} x,
$$

from which we derive the Basic Reproduction Ratio, when $h_{p}=1$ :

$$
\mathcal{R}_{0}^{2}=\frac{\phi^{2} a b}{\left(k_{2}+\gamma\right)\left(\alpha_{v}+\delta\right)} \tilde{\rho}
$$

It is worth making a remark regarding the basic reproduction number in [3] compared to the value in (5). Unlike [3], here the term $-k_{1} l_{p}$ is considered as part of the rate of disease progression. Let $h_{p}^{*}=\frac{\left(k_{2}+\gamma\right)\left(\alpha_{v}+\delta\right)}{\phi^{2} a b \tilde{\rho}}$ such that $\mathcal{R}^{2}\left(h_{p}^{*}\right)=1$. Thus, all plant populations such that $h_{p}<h_{p}^{*}$ verifies $\mathcal{R}^{2}\left(h_{p}\right)<1$. Consider the following subset of $\mathcal{P}$

$$
\mathcal{P}_{s}=\left\{x=\left(h_{p}, 0,0,0\right)^{T}: 0 \leq h_{p} \leq \min \left\{1, h_{p}^{*}\right\}\right\}
$$

Then, according to [39] we deduce

Theorem 2 (Stability). All stable equilibria of the dynamical system (3) belong to $\mathcal{P}_{\text {s. }}$ The equilibria in $\mathcal{P}_{u}=\mathcal{P} \backslash \mathcal{P}_{s}$ are unstable. The set $\mathcal{P}_{s}$ is a stable invariant set with basin of attraction $\Omega \backslash \mathcal{P}_{u}$.

Remark 3. Practically, the previous theorem means that every trajectory initiated in $\Omega \backslash \mathcal{P}_{u}$ converges to a point in $\mathcal{P}_{s}$. There is no analytical formula to estimate $h_{p, \infty}$, the long term healthy plant population.

Remark 4. According to (5), $\phi$, the contact rate, is an interesting parameter to control to reduce the epidemiological risk. For instance, a way to reduce the visits, and thus $\phi$, is to use eco-friendly nets to protect the crops. This technique is under study, for example, in Kenya [37].

We provide some numerical simulations to show the behavior of the system when introducing the disease through the vector or the plants. We consider the following parameters summarized in Table 1 . We also

\begin{tabular}{|l||c|c|c|c|c|c|c|c|c|c|}
\hline Parameter & $\phi$ & $a$ & $b$ & $k_{1}$ & $k_{2}$ & $\alpha_{v}$ & $\mu_{1}$ & $\mu_{2}$ & $\beta$ & $\delta$ \\
\hline Value & 2 & 0.2 & 0.2 & 0.2 & 0.1 & 0.06 & 0.05 & 0.01 & 0.01 & 1 \\
\hline
\end{tabular}

Table 1: Parameters values

consider two cases: without sanitary harvest, $\gamma=0$, and with sanitary harvest, $\gamma=0.02$. As seen in Figs. 2, 3 , and 4 , all trajectories enter the interval $\left[0, h_{p}^{*}\right]$, whatever the initial conditions, where $h_{P}^{*} \approx 0.66\left(h_{P}^{*} \approx 0.79\right.$ when $\gamma=0.02)$ is represented by a circle on the $x$-axis denoting the density of healthy plants. Thus, we recover the theoretical result provided in Theorem 2 .

In Fig. 2, page 7, we consider different levels of initially infected plants. Thus, the final number of infected plants will depend on the initial number of infected plants. As expected, even a small sanitary harvest can have a positive effect on the number of healthy plants at the end of the epidemic: compare Figs. $2 a$ and $2 b$.

If the lifespan of the virus is longer (shorter), i.e. $\delta<(>) 1$, then $h_{p}^{*}$ decreases (increases). This is meaningful since the longer the virus lifespan, the longer the infected pest stays infected which implies that 

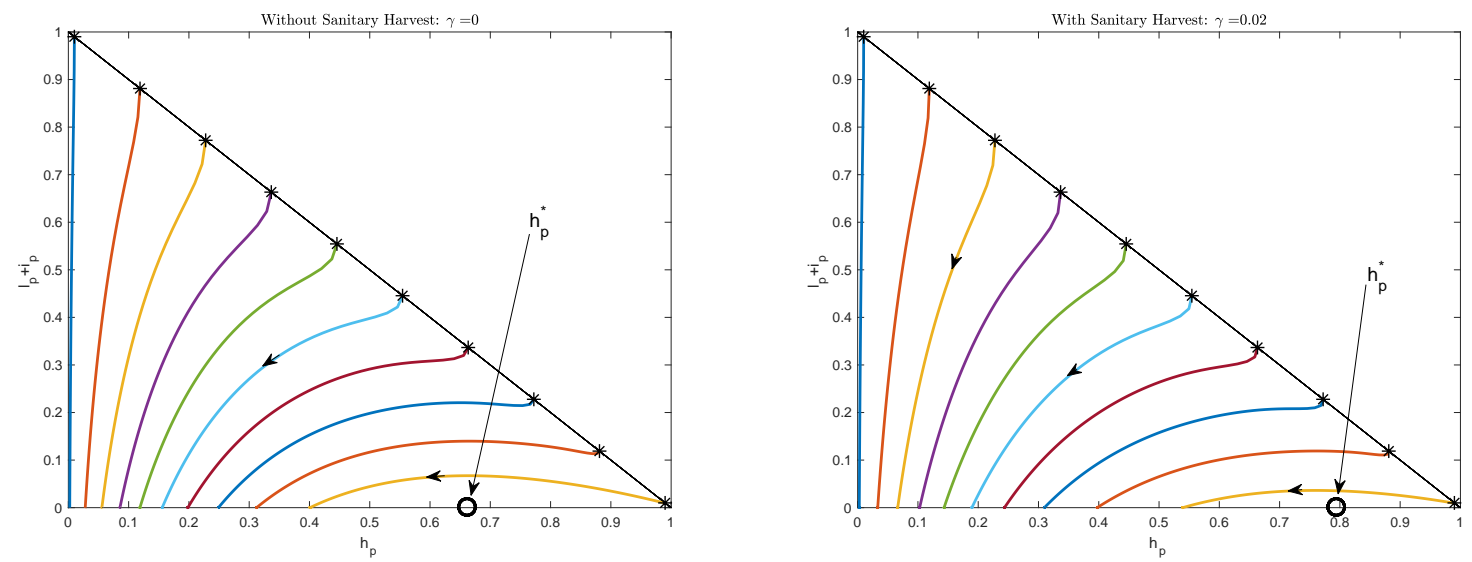

Figure 2: Plants are infected. Simulations without and with sanitary harvest: (a) $\gamma=0$ (b) $\gamma=0.02$
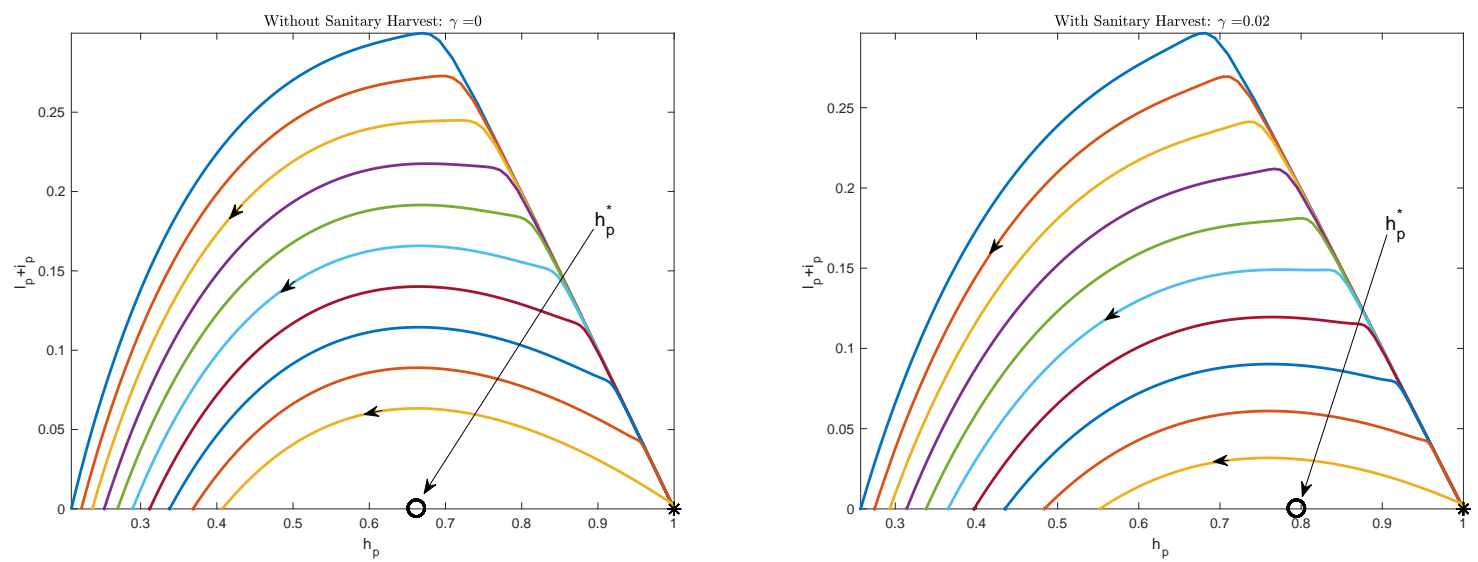

Figure 3: Vectors are infected. Simulations without and with sanitary harvest: (a) $\gamma=0$ (b) $\gamma=0.02$
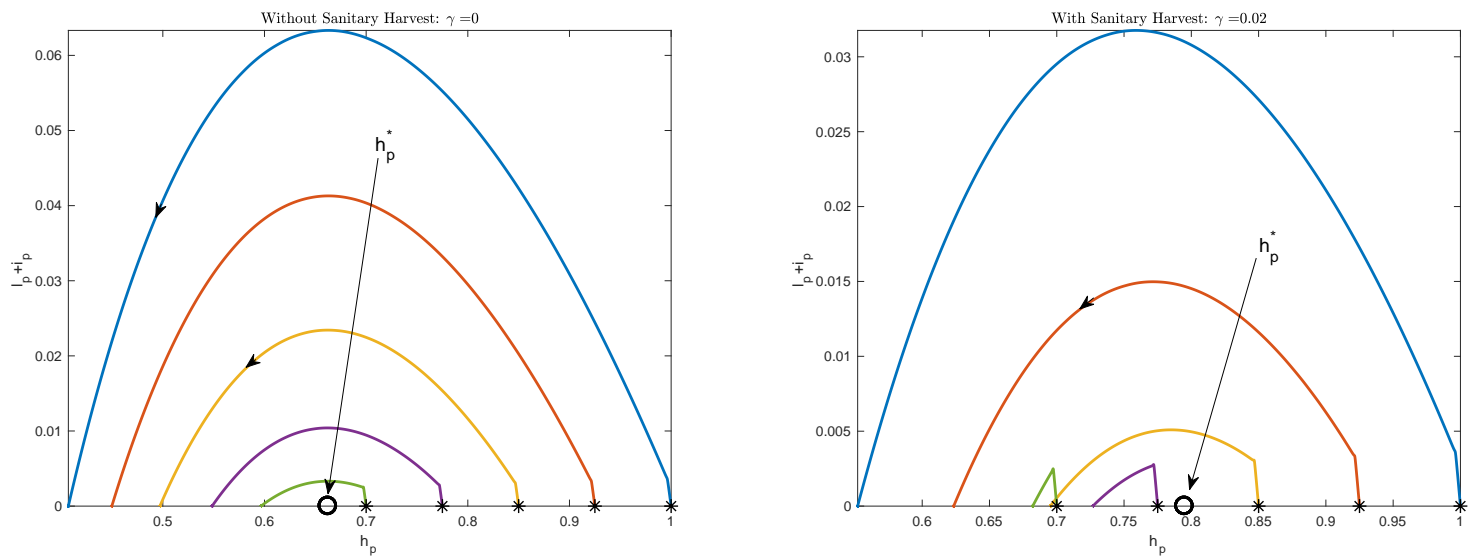

Figure 4: Diluted Effect or the impact of planting a certain percentage of "resistant" plants/seeds 
more plants are infected. In contrary, when the lifespan of the virus is short, then the impact of the epidemic is smaller.

In Fig. 3, page 7, all plants are susceptible $\left(h_{p}=1\right)$. Then, depending on the level of initial infective vectors, the trajectories show that the whole susceptible plant population can be impacted completely.

Finally, in Fig. 4, page 7, if we assume that a certain proportion of the seeds are resistant, then the impact of the disease can be limited. For instance, if the proportion of resistant plants/seeds is about $15 \%$, then, according to the simulation the loss can be estimated around $\frac{85-50}{85} \times 100 \approx 30 \%$ (see the yellow trajectory in Fig. 4 and compare to the yellow trajectory in Fig. 2a, where the loss was almost $60 \%$ ). The use of a certain fraction of resistant plants can be a good strategy to lower the impact of the pest. However, in general resistant seeds are more expensive, and thus a balance should be found between the cost of resistant seeds and crop loss. Other control tools can also be considered as explained in the next subsection. Finally, if we consider resistant plant and sanitary harvest (Fig. 4b, page 7), then $h_{p}^{*}=0.795$ and the loss is around $\frac{85-68}{85} \times 100 \approx 20 \%$ (see the yellow trajectory in Fig. $4 \mathrm{~b}$ ). Clearly a combination of strategies is the best way to reduce the outbreak.

\subsection{Control strategies}

For decades, many control strategies have been developed with mitigated success like for malaria control. For plants diseases, the worst control method is the use of pesticides and it is acknowledged that they are mostly ineffective against aphids. First, because the time needed to transmit the virus is more or less immediate, and thus an infected aphid can transmit the non-persistent virus before being killed by the insecticide. Secondly, the use of pesticides can repell the aphids causing a rapid movement in (non-treated) parts of the crops, and thus causing greater spreading of the diseases.

As shown in the previous figures, infective plant harvest is a way to reduce the impact of the disease. However, it could be possible to choose the harvesting parameter $\gamma=\gamma^{*}$ such that $h_{p}^{*}=1$. For instance, using the same parameters, we found that $\gamma^{*} \approx 0.051$ reduces the loss drastically (compare Fig. 5 with Fig. 2 ). Of course it is possible to choose $\gamma>\gamma^{*}$, but then, probably this is not economically sustainable.

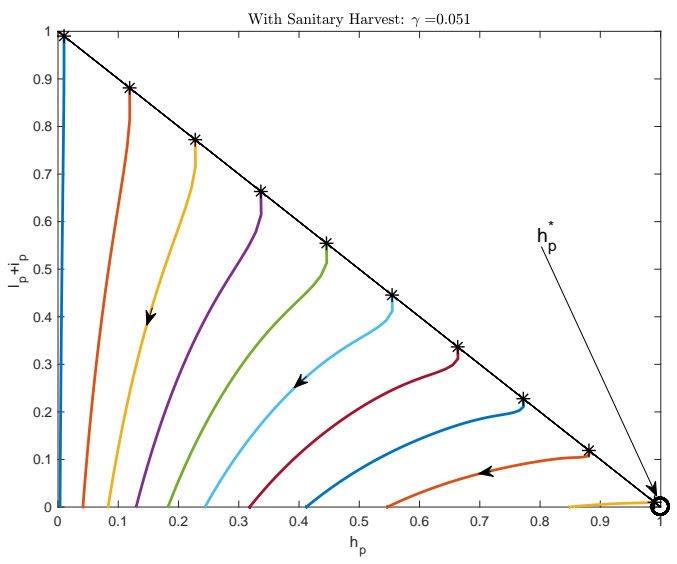

Figure 5: Plants are infected. Simulation with sanitary harvest rate $\gamma=\gamma^{*}$ such that $h_{p}^{*}=1$.

A very interesting and promising technique is the use of barrier plants or secondary plants to protect the main crops from diseases. Here different strategies are possible. For example, border plants, intercrops, mixed cropping, etc. This is a kind of manipulation strategies to reduce the probability of contact between the crops and the aphids. In general, it suffices to consider plants suitable for the aphids (feeding, reproduction) based on good knowledge of their biology/ecology. For example:

- Aphids respond to visual stimuli and in particular visual contrast [10].

- Aphids cannot distinguish host and non-host plants before landing. Then, as explained above, they 
can make exploratory probes with their mouth-parts, such that any virus particle can be released on to non-host plants. Of course, the longer they stay on a non-host plant, the less effective is the virus.

- Loosing time on non-host plants make the aphids less active to search new (host) plants.

It is possible to study the impact of barrier plants, as mixed cropping for instance, using the temporal model developed above. By introducing $K_{b}$, the total amount of barrier plants, then model (1)-(2) is modified with $K$ now replaced by $K+K_{b}$. Using the following change of variables, i.e., $h_{p}=\frac{H_{p}}{K+K_{b}}, l_{p}=\frac{L_{p}}{K+K_{b}}$, $i_{p}=\frac{I_{p}}{K+K_{b}}, r_{p}=\frac{R_{p}}{K+K_{b}}, s_{v}=\frac{S_{v}}{V}, i_{v}=\frac{I_{v}}{V}$, we obtain the same equations as in (3) and (4) except that $\rho=V /\left(K+K_{b}\right)$ and

$$
\frac{d K}{d t}=-\gamma i_{p}\left(K+K_{b}\right),
$$

We set $\tilde{\rho}_{b}=\frac{\alpha_{v}-\mu_{1}}{\mu_{2}\left(K_{\infty}+K_{b}\right)}$, where $K_{\infty}=\lim _{t \rightarrow \infty} K(t)>0$. Straightforward computations of the basic reproduction number in the new setting gives

$$
\mathcal{R}_{0, b}^{2}=\frac{\phi^{2} a b}{\left(k_{2}+\gamma\right)\left(\alpha_{v}+\delta\right)} \tilde{\rho}_{b},
$$

with $\tilde{\rho}_{b}=\frac{\alpha_{v}-\mu_{1}}{\mu_{2}\left(K_{\infty}+K_{b}\right)}$, such that

$$
\mathcal{R}_{0, b}^{2}=\frac{K_{\infty}}{K_{\infty}+K_{b}} \mathcal{R}_{0}^{2} .
$$

Thus obviously the use of barrier plants may have an impact on the basic reproduction number and thus on the number of healthy plants at the end of the epidemic as shown in Fig. 6, page 9.
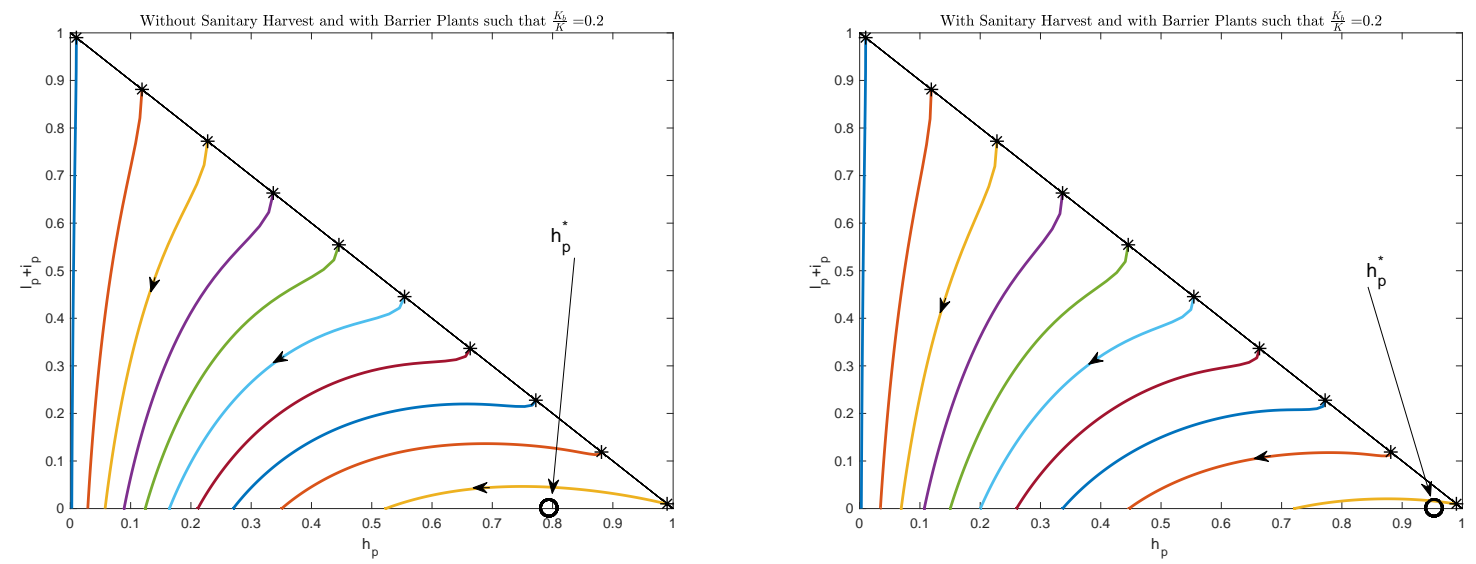

Figure 6: Plants are infected. Simulations with barrier plants without (a) and with (b) sanitary harvest

In Fig. 6, we consider the same computations done in Fig. 2, with $K_{b}=0.2 \times K$. All trajectories enter the interval $\left[0, h_{b}^{*}\right]$, with $h_{b}^{*} \approx 0.795$. It is interesting to notice that after the outbreak, the number of healthy individuals in Fig. 6 is significantly larger compared to healthy individuals in Fig. 2. However, the improvement due to barrier plants is significant if the infection is low. For instance, when $i_{p}=0.01$ (yellow trajectory) the loss is only around $48 \%$ while, in Fig. 2 the loss is around $60 \%$. In contrary, when the initial infective population $i_{p}$ is large, then the benefit of using barrier plant, at least when $\frac{K_{b}}{K}=0.2$ is negligible. If we consider a combination of barrier plant and sanitary harvest, with $\gamma=0.02$, it is possible to improve the results. All trajectories enter the interval $\left[0, h_{b}^{*}\right]$, with $h_{b}^{*} \approx 0.954$. In that case, the loss reduces to $25 \%$.

However, if we consider barrier plants as border plants, model (1)-(2) is far from being realistic. Indeed, according to Fig. 13, page 23, the homogeneous assumptions related to the temporal model is not available, and the space component becomes thereby very important. 
In fact the space component is definitively necessary if we consider the following obvious fact: pests are moving, while plants are not. That is why it is necessary to extend our model using partial differential equations, on the vector components. This is what we intend to develop in the next section.

\section{Towards a more realistic model}

The previous model suffers from a constrained assumption of homogeneous distribution between plants and vectors. While for vectors this assumption is realistic, this is not the case for plants that do not move. In contrary, only vectors can move. Thus we propose to extend our model by introducing a diffusion operator in the vector equations in order to take into account a random dispersal of vectors.

Thus ODEs system (1)-(2) shifts to the following ODEs-PDEs system, for all $(x, t) \in \mathbb{R} \times \mathbb{R}_{+}$

$$
\begin{gathered}
\left\{\begin{array}{l}
\frac{\partial H_{p}}{\partial t}=-\phi b I_{v} \frac{H_{p}}{K} \\
\frac{\partial L_{p}}{\partial t}=\phi b I_{v} \frac{H_{p}}{K}-k_{1} L_{p}, \\
\frac{\partial I_{p}}{\partial t}=k_{1} L_{p}-\left(k_{2}+\gamma\right) I_{p}, \\
\frac{\partial R_{p}}{\partial t}=k_{2} I_{p}, \\
\frac{\partial S_{v}}{\partial t}=D \frac{\partial^{2} S_{v}}{\partial x^{2}}+\alpha_{v} V-\left(\mu_{1}+\mu_{2} V\right) S_{v}-\phi a S_{v} \frac{I_{p}}{K}+\delta I_{v} e^{-\frac{\phi a I_{p}}{\delta K}} \\
\frac{\partial I_{v}}{\partial t}=D \frac{\partial^{2} I_{v}}{\partial x^{2}}+\phi a S_{v} \frac{I_{p}}{K}-\delta I_{v} e^{-\frac{\phi a I_{p}}{\delta K}}-\left(\mu_{1}+\mu_{2} V\right) I_{v},
\end{array}\right.
\end{gathered}
$$

with non-negative initial conditions and appropriate boundary conditions. It is straightforward to observe that the total vector population follows:

$$
\left\{\begin{array}{l}
\frac{\partial V}{\partial t}=D \frac{\partial^{2} V}{\partial x^{2}}+\alpha_{v} V-\left(\mu_{1}+\mu_{2} V\right) V, \quad(x, t) \in \mathbb{R} \times \mathbb{R}_{+} \\
V(x, 0)=V_{0}(x), \quad x \in \mathbb{R} .
\end{array}\right.
$$

We recognized the well-known Fisher's reaction diffusion equation, for which we consider the following boundary conditions $V(-\infty, t)=V^{*}$ and $V(+\infty, t)=0$ Let us recall that a traveling-wave solution of (11) is defined as $V(x, t)=v(x-c t)=v(z)$, where $z=x-c t$ and $c \in \mathbb{R}$ is called the traveling-wave speed. The function $v$ is solution of

$$
v^{\prime \prime}+\frac{c}{D} v^{\prime}+\frac{\alpha_{v}-\mu_{1}}{D}\left(1-\frac{v}{V^{*}}\right) v=0 .
$$

Using the notation of [40], a $\left[v_{1}, v_{2}\right]$-traveling wave is a solution of (12) such that

$$
\lim _{z \rightarrow-\infty} v(z)=v_{1}, \quad \text { and } \quad \lim _{z \rightarrow+\infty} v(z)=v_{2}
$$

If in addition $v_{1}$ or $v_{2}$ is stable while the other is unstable, then the $\left[v_{1}, v_{2}\right]$-traveling wave is called a mono-stable traveling wave. For sake of simplicity, we will use this notation in the rest of the paper.

Assuming $\mathcal{N}>1$, the following results hold [40]

- Two homogeneous equilibria exist: 0 and $V^{*}=\frac{\alpha_{v}-\mu_{1}}{\mu_{2}}$.

- The equilibrium 0 is unstable, while $V^{*}$ is asymptotically stable. 
- For all $c \geq c_{v}^{*}$, where

$$
c_{v}^{*}=2 \sqrt{D\left(\alpha_{v}-\mu_{1}\right)}=2 \sqrt{D \mu_{1}(\mathcal{N}-1)} .
$$

Equation (11) admits a monotone mono-stable $\left[V^{*}, 0\right]$-wave $[16,40]$.

Taking into account the dynamics of $V$, we expect the following behavior of the solutions at infinity

$$
\begin{array}{r}
\lim _{x \rightarrow-\infty} H_{p}(x, t)=H_{p, \infty}, \quad \lim _{x \rightarrow+\infty} H_{p}(x, t)=K_{H}, \\
\lim _{x \rightarrow \pm \infty} L_{p}(x, t)=0, \\
\lim _{x \rightarrow-\infty} R_{p}(x, t)=R_{p, \infty}, \quad I_{p}(x, t)=0, \\
\lim _{x \rightarrow+\infty} H_{p}(x, t)=K_{R},
\end{array}
$$

where $H_{p, \infty}$ and $R_{p, \infty}$ are unknown non negative constants, and $K_{H}+K_{R}=K$.

System (9)-(10) can be rewritten as follows

$$
\left\{\begin{array}{l}
\mathbf{u}_{t}-D \mathbf{u}_{x x}=f(\mathbf{u}, \mathbf{v}) \\
\mathbf{u}_{t}=g(\mathbf{u}, \mathbf{v})
\end{array}\right.
$$

where $D>0$ and

$$
\begin{gathered}
\mathbf{u}=\left(\begin{array}{c}
S_{v} \\
I_{v}
\end{array}\right), \quad \mathbf{v}=\left(\begin{array}{c}
H_{p} \\
L_{p} \\
I_{p} \\
R_{p}
\end{array}\right), \\
f(\mathbf{u}, \mathbf{v})=\left(\begin{array}{c}
\alpha V-\left(\mu_{1}+\mu_{2} V\right) S_{v}+\delta I_{v} e^{-\frac{\phi a I_{p}}{\delta K}}-\frac{\phi a I_{p}}{K} S_{v} \\
-\left(\mu_{1}+\mu_{2} V\right) I_{v}-\delta I_{v} e^{-\frac{\phi a I_{p}}{\delta K}}+\frac{\phi a I_{p}}{K} S_{v}
\end{array}\right), \quad g(\mathbf{u}, \mathbf{v})=\left(\begin{array}{c}
-\frac{\phi a I_{v}}{K} H_{p} \\
\frac{\phi a I_{v}}{K} H_{p}-k_{1} L_{p} \\
k_{1} L_{p}-\left(k_{2}+\gamma\right) I_{p} \\
k_{2} I_{p}
\end{array}\right) .
\end{gathered}
$$

We derive briefly some results related system (9)-(10). First, we consider the following spaces

$$
\mathcal{S}=\left\{(u, v) \mid 0 \leq S_{v}, I_{v} \in L^{2}(\mathbb{R}) ; 0 \leq H_{p}, L_{p}, I_{p}, R_{p} \in L^{\infty}(\mathbb{R})\right\},
$$

and

$$
\mathcal{S}_{K, V^{*}}=\left\{(u, v) \in \mathcal{S} \mid S_{v}+I_{v} \leq V^{*} ; H_{p}+L_{p}+I_{p}+R_{p} \leq K\right\} .
$$

System (17) is a partly dissipative or partially degenerate system for which several works have been done (see for instance $[29,21]$ ).

Theorem 5 (Existence - uniqueness). For any initial values $\left(u_{0}, v_{0}\right) \in \mathcal{S}_{K, V^{*}}$, system (17) admits a unique non-negative bounded solution such that

$$
\begin{aligned}
& u \in\left(C\left([0, \infty) ; L^{\infty}(\mathbb{R})\right) \cap C^{1}\left((0, \infty) ; L^{\infty}(\mathbb{R})\right)\right)^{4}, \\
& v \in\left(C\left([0, \infty) ; L^{\infty}(\mathbb{R})\right) \cap C\left([0, \infty) ; H^{2}(\mathbb{R})\right) \cap C^{1}\left([0, \infty) ; L^{2}(\mathbb{R})\right)\right)^{2} .
\end{aligned}
$$

Proof. To show (global) existence, we first need to show local existence and then give a priori estimates. Local existence and uniqueness can be obtained using [33, Theorem 2.1] (see also [35, Theorem 1, page 111]). A priori $L^{\infty}$ estimates are also relatively straightforward to obtain. Then, $F=\left(\begin{array}{c}f(u, v) \\ g(u, v)\end{array}\right)$ being quasi-positive (i.e. $\zeta=\left(\zeta_{i}\right)_{i=1}^{n} \in \mathbf{R}_{+}^{n}$ and $\zeta_{k}=0 \Rightarrow F_{k}(\zeta) \geq 0$ ) and the maximum principle imply that the solution remains nonnegative and bounded.

Remark 6. Notice that system (9)-(10) is very close to the systems studied in [21] (except the boundary conditions, but as stated in [21] only few modifications in the proof lead to the same existence result).

In addition, it is possible and straightforward to study the long term behavior of the model. Indeed, the solution being non-negative and bounded for all $x \in[0, L]$, and $t \geq 0$, we have 
- $\lim _{t \rightarrow \infty} H_{p}(x, t)=H_{p}^{\infty}(x)$,

- $\lim _{t \rightarrow \infty} L_{p}(x, t)=0$,

- $\lim _{t \rightarrow \infty} I_{p}(x, t)=0$,

- $\lim _{t \rightarrow \infty} R_{p}(x, t)=R_{p}^{\infty}(x)$,

and

- $S_{v}(x, t)$ converges to $V^{*}$ in $L^{2}(\mathbb{R})$ and $H_{1}(\mathbb{R})$,

- $I_{v}(x, t)$ converges to 0 in $L^{2}(\mathbb{R})$ and $H_{1}(\mathbb{R})$.

According to equation $(9)_{4}, \frac{\partial R_{p}}{\partial t}=k_{2} I_{p} \geq 0$, and, since $R_{p}$ is bounded by $K$, we deduce that $R(x, t)$ converges to some limit $R_{P}^{\infty}(x)$ as $t$ goes to $\infty$, which also implies that $I_{P}(x, t) \in L^{1}(0, \infty)$ for all $x \in[0, L]$. Using $(9)_{4}$ and $(9)_{3}$ such that

$$
\frac{\partial I_{p}}{\partial t}+\frac{k_{2}+\gamma}{k_{2}} \frac{\partial R_{p}}{\partial t}=k_{1} L_{p} \geq 0
$$

implies that $I_{P}+\frac{k_{2}+\gamma}{k_{2}} R_{p}$ converges to some limit, which implies that $I_{p}(x, t)$ converges to some limit $I_{p, \infty}$. Since $I_{P}(x, t) \in L^{1}(0, \infty)$ for all $x$, this implies necessarily that $I_{p, \infty}(x)=0$ for all $x$.

Finally, since $\frac{\partial H_{p}}{\partial t} \leq 0$, we deduce that $H_{p}(x, t)$ converges to $H_{p}^{\infty}(x)$ too. Summing $(9)_{1}$ and $(9)_{2}$, we deduce that $H_{p}(x, t)+L_{p}(x, t)$ converges to some limit too as $t$ goes to infinity, and in particular, $L_{p}(x, t)$ converges to some limit, $L_{p, \infty}(x)$ too. Using equation such that $H_{p}^{\infty}(x)+R_{p}^{\infty}(x) \leq K$ for all $x \in[0, L]$.

Multiplying equation $(10)_{2}$ by $I_{v}$ and integrating, leads to the following inequality

$$
\frac{1}{2} \frac{d}{d t}\left\|I_{v}\right\|_{2}^{2}+D\left\|\nabla I_{v}\right\|_{2}^{2}+\mu_{1}\left\|I_{v}\right\|_{2}^{2} \leq C\left\|I_{p}\right\|_{2}^{2} .
$$

Using the fact that $\lim _{t \rightarrow \infty} \int_{0}^{L} I_{p}^{2}(x, t) d x=0$, we deduce that $\lim _{t \rightarrow \infty}\left\|I_{v}(x, t)\right\|_{2}=0$. Similarly, multiplying equation $(10)_{2}$ by $\frac{\partial I_{v}}{\partial x}$, we deduce $\lim _{t \rightarrow \infty}\left\|\frac{\partial I_{v}}{\partial x}(x, t)\right\|_{2}=0$. Finally, using the fact that $V=S_{v}+I_{v}$, we deduce that $S_{v}(x, t)$ converges to $V^{*}$ in $L^{2}(\mathbb{R})$ and in $H^{1}(\mathbb{R})$

Remark 7. When $\gamma=0, H_{p}^{\infty}$ and $R_{p}^{\infty}$ verify $H_{p}^{\infty}(x)+R_{p}^{\infty}(x)=K$ for all $x \in[0, L]$.

It would be nice to be able to go further in the qualitative study on system (9)-(10). In particular, studying the existence or not of traveling wave solutions, that are particular solutions of the form $\phi(x-c t)$, where $c$ is the traveling wave speed. Different tools may exist [40] to study this system. However, as a first step, we propose to consider and study reduced or sub-models of system (9)-(10) in order to show that, traveling-wave solutions may exist. In addition, in some particular cases, we are able to obtain some information about pulse or front traveling-waves.

\section{A useful and illustrative subsystem}

Assuming that plants have only two epidemiological states, i.e., $H_{p}$ and $I_{p}$ such that $H_{p}+I_{p}=K=$ constant, with $\gamma=0$, system (9)-(10) reduces to

$$
\left\{\begin{array}{l}
\frac{\partial I_{p}}{\partial t}=b \phi\left(1-\frac{I_{p}}{K}\right) I_{v} \\
\frac{\partial I_{v}}{\partial t}=D \frac{\partial^{2} I_{v}}{\partial x^{2}}+\phi a\left(V-I_{v}\right) \frac{I_{p}}{K}-\left(\delta e^{-\frac{\phi a I_{p}}{\delta K}}+\left(\mu_{1}+\mu_{2} V\right)\right) I_{v} \\
\frac{\partial V}{\partial t}=D \frac{\partial^{2} V}{\partial x^{2}}+\alpha_{v} V-\left(\mu_{1}+\mu_{2} V\right) V .
\end{array}\right.
$$


System (18) has a unique solution, and admits the following trivial equilibria $(0,0,0),\left(0,0, V^{*}\right),(K, 0,0)$, $\left(I_{p}^{*}, 0,0\right)$, where $I_{p}^{*}>0$. It is straightforward to show that $(0,0,0),(K, 0,0),\left(0,0, V^{*}\right)$, and $\left(I_{p}^{*}, 0,0\right)$ are all unstable. For all $x \in[0, L]$, system (18) leads to

$$
\lim _{t \rightarrow \infty} I_{p}(x, t)=K, \quad \lim _{t \rightarrow \infty} I_{v}(x, t)=I_{v}^{\infty}(x),
$$

and it is possible to estimate $I_{v}^{\infty}$. If we assume that all plants are infected or if $t$ is large enough such that $I_{p}(x, t)=K$, and $V(x, t)=V^{*}$, then the previous system becomes

$$
\frac{\partial I_{v}}{\partial t}=D \frac{\partial^{2} I_{v}}{\partial x^{2}}+\phi a\left(V^{*}-I_{v}\right)-\left(\delta e^{-\frac{\phi a}{\delta}}+\alpha_{v}\right) I_{v},
$$

which admits only one homogeneous solution

$$
I_{v}^{\infty}=\frac{\phi a}{\phi a+\delta e^{-\phi a / \delta}+\alpha_{v}} V^{*} .
$$

In addition, as $\delta$ increases, $I_{v}^{\infty}$ decreases with a lower limit being $\frac{\phi a}{\phi a+\delta+\alpha_{v}} V^{*}$

Since (18) $)_{3}$ admits a traveling wave solution (up to a translation), it seems reasonable to expect the same properties for $(18)_{1,2}$.

It is relatively obvious to show that system (18) is a (partially degenerate or partly dissipative) monotone system since some but not all diffusion coefficients are zero. For this type of (monotone) system, general result of existence of traveling-wave solution are available, in particular in [40]. In fact system (18) can be rewritten as follows

$$
\left\{\begin{array}{l}
\frac{\partial u}{\partial t}=\mathbf{D} u+\mathbf{G}(u), \forall(x, t) \in[0, L] \times(0, T), \\
u(0, x)=u_{0}(x)
\end{array}\right.
$$

with $\mathbf{D}=\operatorname{diag}(0, D, D)$ and

$$
\mathbf{G}(u)=\left(\begin{array}{c}
b \phi\left(1-\frac{I_{p}}{K}\right) I_{v} \\
\phi a\left(V-I_{v}\right) \frac{I_{p}}{K}-\left(\delta e^{-\frac{\phi a I_{p}}{K \delta}}+\alpha_{v}\right) I_{v} \\
\left(\alpha_{v}-\mu_{1}\right) V\left(1-\frac{V}{V^{*}}\right)
\end{array}\right)
$$

Let us study the following two sub-cases that will be helpful to deduce some dynamics of system (18). This will be done in Section 3.3. However, we can already observe in Figs. 8, page 20, that traveling waves exist for this system.

\subsection{Assuming that all plants are already Infected}

This is equivalent to consider that $I_{p}=K$, which implies that system (18) reduces to

$$
\left\{\begin{array}{l}
\frac{\partial I_{v}}{\partial t}=D \frac{\partial^{2} I_{v}}{\partial x^{2}}+\phi a\left(V-I_{v}\right)-\left(\delta e^{-\frac{\phi a}{\delta}}+\left(\mu_{1}+\mu_{2} V\right)\right) I_{v}, \\
\frac{\partial V}{\partial t}=D \frac{\partial^{2} V}{\partial x^{2}}+\alpha_{v} V-\left(\mu_{1}+\mu_{2} V\right) V .
\end{array}\right.
$$

We recover a standard reaction-diffusion problem, with only two homogeneous equilibria $(0,0)$ and $\left(I_{v}^{\infty}, V^{*}\right)$. It is also worth to note that $(0,0)$ is unstable, while $\left(I_{v}^{\infty}, V^{*}\right)$ is globally asymptotically stable. In fact, setting $\mathbf{u}=\left(i_{v}, v\right)=\left(\frac{I_{v}}{V^{*}}, \frac{V}{V^{*}}\right)$, system $(21)$ can be rewritten as follows

$$
\left\{\begin{array}{l}
\frac{\partial i_{v}}{\partial t}=D \frac{\partial^{2} i_{v}}{\partial x^{2}}+\left(\phi a-\left(\alpha_{v}-\mu_{1}\right) i_{v}\right) v-\left(\phi a+\delta e^{-\frac{\phi a}{\delta}}+\mu_{1}\right) i_{v} \\
\frac{\partial v}{\partial t}=D \frac{\partial^{2} v}{\partial x^{2}}+\left(\alpha_{v}-\mu_{1}\right) v(1-v)
\end{array}\right.
$$


where we have used $V^{*}=\frac{\alpha_{v}-\mu_{1}}{\mu_{2}}$. Setting $\mathbf{u}=\left(i_{v}, v\right)$ in (22), the system can be rewritten as follows

$$
\left\{\begin{array}{l}
\frac{\partial \mathbf{u}}{\partial t}=\mathbf{D} \frac{\partial^{2} \mathbf{u}}{\partial x^{2}}+\mathbf{F}(\mathbf{u}), \forall(x, t) \in[0, L] \times(0, T), \\
\mathbf{u}(0, x)=\mathbf{u}_{0}(x)
\end{array}\right.
$$

with

$$
\mathbf{D}=\left(\begin{array}{cc}
D & 0 \\
0 & D
\end{array}\right) \quad \text { and } \quad \mathbf{F}(\mathbf{u})=\left(\begin{array}{c}
\left(\phi a-\left(\alpha_{v}-\mu_{1}\right) i_{v}\right) v-\left(\phi a+\delta e^{-\frac{\phi a}{\delta}}+\mu_{1}\right) i_{v} \\
\left(\alpha_{v}-\mu_{1}\right) v(1-v)
\end{array}\right)
$$

Since $i_{v}(\cdot, t) \leq i_{v}^{\infty}<\frac{\phi a}{\alpha_{v}-\mu_{1}}$, where

$$
i_{v}^{\infty}=\frac{\phi a}{\phi a+\delta e^{-\phi a / \delta}+\alpha_{v}}
$$

for all $t \geq 0$, we can easily deduce that system (23)-(24) is monotone cooperative. Recall that a reactiondiffusion system, like system (23), is said to be cooperative if $\mathbf{F}$ is differentiable and the matrix $\mathbf{F}^{\prime}(u)$ is cooperative, that is all off-diagonal entries are non-negative. Then, we can show

Theorem 8. System (23)-(24) admits a mono-stable travelling-wave solution with single spreading speed $c_{v}^{*}$.

Proof. We apply [26, Theorem 4.2]. In particular, we have to verify the following main assumptions:

Assumptions (H) (Theorem 4.2 in [26])

1. $\mathbf{F}(\mathbf{0})=\mathbf{0}$ and there is a $\beta \gg 0$ such that $\mathbf{F}(\beta)=0$, which is minimal in the sense that there is no $\mathbf{w}$ other than $\mathbf{0}$ and $\beta$ such that $\mathbf{F}(\mathbf{w})=0$, and $0 \ll \mathbf{w} \leq \beta$.

2. System (20) is cooperative; i.e., $\mathbf{F}_{i}(\mathbf{u})$ is non decreasing in all components of $u$ with the possible exception of the $i$ th one.

3. $\mathbf{F}$ does not depend explicitely on $x$ and $t$, and the diagonal terms of $\mathbf{D}$ are constant.

4. $\mathbf{F}(\mathbf{p})$ is continuous and piecewise differentiable in $\mathbf{p}$ for $\mathbf{0} \leq \mathbf{p} \leq \beta$ and differentiable at $\mathbf{0}$. The Jacobian matrix $\mathbf{F}^{\prime}(\mathbf{0})$, whose off-diagonal entries are non-negative, has a positive eigenvalue whose eigenvectors has positive components.

5. The mobilities $d_{i}$, which are the diagonal and only non-zero entries of $\mathbf{D}$ are positive.

$\mathbf{F}(\mathbf{u})$, as it is defined in (24), vanishes only at points $\mathbf{0}=(0,0)$ and $\beta=\left(i_{v}^{\infty}, 1\right)$. In addition $\mathbf{F}$ is differentiable, with

$$
\mathbf{F}^{\prime}(\mathbf{u})=\left(\begin{array}{cc}
-\left(\phi a+\delta e^{-\frac{\phi a}{\delta}}+\mu_{1}+\left(\alpha_{v}-\mu_{1}\right) v\right) & \left(\phi a-\left(\alpha_{v}-\mu_{1}\right) i_{v}\right) \\
0 & \left(\alpha_{v}-\mu_{1}\right)(1-2 v)
\end{array}\right) .
$$

Clearly $\mathbf{F}$ and $\mathbf{D}$, as defined in (24) verify Assumptions $(\mathrm{H})$. Let us just check assumption $(\mathrm{H})_{4}$. Since such that

$$
\mathbf{F}^{\prime}(\mathbf{0})=\left(\begin{array}{cc}
-\left(\phi a+\delta e^{-\frac{\phi a}{\delta}}+\mu_{1}\right) & \phi a \\
0 & \left(\alpha_{v}-\mu_{1}\right)
\end{array}\right),
$$

$\mathbf{F}^{\prime}(\mathbf{0})$ admits a positive eigenvalue $\alpha_{v}-\mu_{1}$, where the related eigenvector is $e_{v}=\left(\frac{\phi a}{\phi a+\alpha_{v}+\delta e^{-\phi a / \delta}}, 1\right)^{T}$, which has positive components. Thus according to Theorem $4.2[26],(22)$ admits a traveling wave solution with a minimum speed $\bar{c}=2 \sqrt{D\left(\alpha_{v}-\mu_{1}\right)}$ that is exactly the speed $c_{v}^{*}$, defined in (13), page 11 .

Remark 9. In fact, it would have been possible to make some analogy between system (22) and system (2.2) studied in [25], to prove theorem 8.

Remark 10. In other words, according to Theorem 8, when the crop is already infected, whatever the values taken by the virus death rate, $\delta$, and the contact rate, $\phi$, the traveling waves of the infected spread like the total population. This result is confirmed by the numerical simulations (see Figs. 7, page 19). 


\subsection{Assuming that the vectors are already established in the crop}

Assuming the vector is already established is equivalent to taking $V=V^{*}$ and we would like to know the effect of introducing an infected vector. Thus system (18) becomes

$$
\left\{\begin{array}{l}
\frac{\partial I_{p}}{\partial t}=b \phi\left(1-\frac{I_{p}}{K}\right) I_{v} \\
\frac{\partial I_{v}}{\partial t}=D \frac{\partial^{2} I_{v}}{\partial x^{2}}+\phi a\left(V^{*}-I_{v}\right) \frac{I_{p}}{K}-\left(\delta e^{-\frac{\phi a I_{p}}{\delta K}}+\alpha_{v}\right) I_{v} .
\end{array}\right.
$$

Here, we have a partially degenerate reaction diffusion system, for which the theorem, used in the previous section, does not work. Instead, we will use results from [13]. First we recall some of the main results in the appendix.

Setting $i_{p}=\frac{I_{p}}{K}$ and $i_{v}=\frac{I_{v}}{V^{*}}$, leads to the following system

$$
\left\{\begin{array}{l}
\frac{\partial i_{p}}{\partial t}=\frac{b \phi V^{*}}{K}\left(1-i_{p}\right) i_{v} \\
\frac{\partial i_{v}}{\partial t}=D \frac{\partial^{2} i_{v}}{\partial x^{2}}+\phi a\left(1-i_{v}\right) i_{p}-\left(\delta e^{-\frac{\phi a i_{p}}{\delta}}+\alpha_{v}\right) i_{v} .
\end{array}\right.
$$

With $u=\left(i_{p}, i_{v}\right)$ and

$$
\mathbf{D}=\left(\begin{array}{cc}
0 & 0 \\
0 & D
\end{array}\right) \quad \text { and } \quad \mathbf{F}(\mathbf{u})=\left(\begin{array}{c}
\frac{b \phi V^{*}}{K}\left(1-i_{p}\right) i_{v} \\
\phi a\left(1-i_{v}\right) i_{p}-\left(\delta e^{-\frac{\phi a_{p}}{\delta}}+\alpha_{v}\right) i_{v}
\end{array}\right)
$$

the previous system (26) can be rewritten as follows

$$
\left\{\begin{array}{l}
\frac{\partial \mathbf{u}}{\partial t}=\mathbf{D u}+\mathbf{F}(\mathbf{u}), \forall(x, t) \in[0, L] \times(0, T), \\
\mathbf{u}(0, x)=\mathbf{u}_{0}(x)
\end{array}\right.
$$

Obviously $\mathbf{F}$ is continuous and differentiable. In addition system (29) admits only two equilibria: $\mathbf{0}=(0,0)$ and $\mathbf{1}=\left(1, i_{v}^{\infty}\right)$. We have the following result

Theorem 11. System (29)-(28) admits a traveling-wave solution $u(x-c t)$, for all $c \geq \bar{c}$, connecting 1 and 0 .

Proof. We apply [13, Theorem 3.1], that is recalled in the appendix (see Theorem 15, page 27), as well as the assumptions (K), page 26. Obviously we have

$$
\frac{\partial \mathbf{F}_{i}}{\partial u_{j}} \geq 0, \quad i, j=1,2 \quad \text { and } \quad i \neq j
$$

which implies that (29) is a monotone and cooperative system [36]. It is also straightforward to show that the solution $u$ verifies $\mathbf{0} \leq u \leq \mathbf{1}$, such that $\mathbf{0}$ and $\mathbf{1}$ are the only points that verify $\mathbf{F}(\mathbf{0})=\mathbf{F}(\mathbf{1})=0$. We have

$$
\mathbf{F}^{\prime}(\mathbf{0})=\left(\begin{array}{ll}
0 & \frac{b \phi V^{*}}{K} \\
\phi a & -\left(\alpha_{v}+\delta\right)
\end{array}\right)
$$

such that $\operatorname{trace}\left(F^{\prime}(\mathbf{0})\right)<0$ and $\operatorname{det}\left(F^{\prime}(\mathbf{0})\right)<0$, which implies that necessarily one eigenvalue is real and positive, such that the stability modulus of $\mathbf{F}^{\prime}(\mathbf{0}), s\left(\mathbf{F}^{\prime}(\mathbf{0})\right.$, is strictly positive. In addition, since $\frac{a b \phi^{2} V^{*}}{K}>0$, $F^{\prime}(\mathbf{0})$ is irreducible. 
Remark 12. If we consider the same problem, but with sanitary harvest, $\gamma>0$ in the $i_{p}$ compartment, then $F^{\prime}(\mathbf{0})$ becomes

$$
F^{\prime}(\mathbf{0})=\left(\begin{array}{ll}
-\gamma & \frac{b \phi V^{*}}{K} \\
\phi a & -\left(\alpha_{v}+\delta\right)
\end{array}\right)
$$

Thus in order to verify $\operatorname{det}\left(F^{\prime}(\mathbf{0})\right)<0$, we have to verify $\gamma\left(\alpha_{v}+\delta\right)-\frac{a b \phi^{2} V^{*}}{K}<0$ or equivalently $\mathcal{R}_{0}=$ $\frac{a b \phi^{2}}{\gamma\left(\alpha_{v}+\delta\right)} \frac{V^{*}}{K}>1$, which is a necessary condition to have $\mathbf{0}$ unstable for the corresponding ODE model. Here, since we consider constant values for the parameters, the reaction-diffusion model and the temporal model have the same Basic Reproduction Number.

Following [13], $\lambda_{A}(\mu)=s\left(A_{\mu}\right)$ is a simple eigenvalue of $A(\mu)$, where $A(\mu)=\mu^{2} \mathbf{D}+\mathbf{F}^{\prime}(\mathbf{0})$, that is

$$
A(\mu)=\left(\begin{array}{ll}
0 & \frac{b \phi V^{*}}{K} \\
\phi a & D \mu^{2}-\left(\alpha_{v}+\delta\right)
\end{array}\right) .
$$

The related characteristic polynomial is given by

$$
p(\lambda)=\lambda\left(\lambda-\left(D \mu^{2}-\left(\alpha_{v}+\delta\right)\right)\right)-\frac{a b \phi^{2} V^{*}}{K},
$$

such that we are able to deduce

$$
\lambda_{A}(\mu)=\frac{1}{2}\left(D \mu^{2}-\left(\alpha_{v}+\delta\right)+\sqrt{\left(D \mu^{2}-\left(\alpha_{v}+\delta\right)\right)^{2}+4 \frac{a b \phi^{2} V^{*}}{K}}\right) .
$$

with a positive eigenvector $v(\mu)=\left(1, \frac{K \lambda_{A}(\mu)}{b \phi V^{*}}\right)^{T}$. Then, let us consider the function $\Phi(\mu)=\frac{\lambda_{A}(\mu)}{\mu}>0$ and we set $\bar{c}=\inf _{\mu>0} \phi(\mu)$. We want to show that the infimum $\bar{c}$ is reached for a positive value $\bar{\mu}$.

Let us briefly study $\Phi(\mu)$ :

$$
\Phi^{\prime}(\mu)=\frac{1}{\mu^{2}}\left(\mu \lambda^{\prime}(\mu)-\lambda(\mu)\right)=\frac{1}{2 \mu^{2}}\left(D \mu^{2}+\left(\delta+\alpha_{v}\right)-\frac{4 \frac{a b \phi^{2} V^{*}}{K}-\left(\alpha_{v}+\delta\right)\left(D \mu^{2}-\left(\alpha_{v}+\delta\right)\right)}{\sqrt{\left(D \mu^{2}-\left(\alpha_{v}+\delta\right)\right)^{2}+4 \frac{a b \phi^{2} V^{*}}{K}}}\right),
$$

such that looking for the zeros of $\Phi^{\prime}$ is equivalent to looking for the zeros of $\mu \lambda^{\prime}(\mu)-\lambda(\mu)$. Setting $x=D \mu^{2}$, and after some computations, this is equivalent to finding the roots of the following fourth order polynomial

$$
p(x)=x^{4}+\left(c-3 b^{2}\right) x^{2}+\left(4 b c+2 b^{3}\right) x-\left(c^{2}+c b^{2}\right),
$$

where $b=\alpha_{v}+\delta$ and $c=4 \frac{a b \phi^{2} V^{*}}{K}$. Note also that the constant coefficient of $p$ is always negative. It is possible to use Descartes rules of sign, to deduce either that $p$ has only positive root when $c>3 b^{2}$, either that $p$ has one or three positive roots when $c<3 b^{2}$.

Thus, we deduce that there exists $x^{*}$, a positive real, such that $\Phi$ reaches its minimum, which implies that $\bar{c}$ is reached at $\bar{\mu}=\sqrt{\frac{x^{*}}{D}}$.

Remark 13. In Fig. 8(b), page 20, we show that the infected front can travel faster than the total Pest front when $\delta$ is small enough (the virus being (semi-) persistent and circulative).

Let us now verify assumption $(\mathrm{K})_{4}$ and $(\mathrm{K})_{5}$, page 26 . Using the fact that $-i_{p} i_{v} \geq-\frac{1}{2}\left(i_{p}^{2}+i_{v}^{2}\right)=$ $-\frac{1}{2}\|\mathbf{u}\|_{2}^{2}$, we deduce

$$
\mathbf{F}(\mathbf{u})=\left(\begin{array}{c}
\frac{b \phi V^{*}}{K}\left(1-i_{p}\right) i_{v} \\
\phi a\left(1-i_{v}\right) i_{p}-\left(\delta e^{-\frac{\phi a_{p}}{\delta}}+\alpha_{v}\right) i_{v}
\end{array}\right) \geq\left(\begin{array}{cc}
0 & \frac{b \phi V^{*}}{K} \\
\phi a & -\left(\alpha_{v}+\delta\right)
\end{array}\right) \mathbf{u}-\alpha_{v}\|\mathbf{u}\|_{2}^{2} \mathbf{1}
$$


with $\alpha_{v}=\frac{1}{2} \max \left\{\frac{b \phi V^{*}}{K} ; \phi a\right\}>0, \sigma=2>1$, for $\mathbf{0}<\mathbf{u}<\mathbf{r}=\mathbf{1}$.

Let us compute

$$
\mathbf{F}(\mathbf{1})=\left(\begin{array}{c}
0 \\
-\left(\delta e^{-\frac{\phi a}{\delta}}+\alpha_{v}\right)
\end{array}\right), \quad \text { and } \quad \mathbf{F}(\rho v(\mu))=\left(\begin{array}{c}
(1-\rho) \lambda_{A}(\mu) \rho \\
\phi a\left(1-\rho \frac{K \lambda_{A}(\mu)}{b \phi V^{*}}\right) \rho-\left(\delta e^{-\frac{\phi a}{\delta} \rho}+\alpha_{v}\right) \frac{K \lambda_{A}(\mu)}{b \phi V^{*}} \rho
\end{array}\right) .
$$

We have

$$
\begin{aligned}
(1-\rho) \lambda_{A}(\mu) \rho & \leq \lambda_{A}(\mu) \rho, \\
\phi a\left(1-\rho \frac{K \lambda_{A}(\mu)}{b \phi V^{*}}\right) \rho-\left(\delta e^{-\frac{\phi a}{\delta} \rho}+\alpha_{v}\right) \frac{K \lambda_{A}(\mu)}{b \phi V^{*}} \rho & =\phi a-\left(\delta e^{-\frac{\phi a}{\delta} \rho}+a \phi \rho+\alpha_{v}\right) \frac{K \lambda_{A}(\mu)}{b \phi V^{*}} \rho .
\end{aligned}
$$

A direct calculation shows that for all $x>0$, we have

$$
\delta e^{-x / \delta}+x>\delta,
$$

which implies that

$$
\phi a-\left(\delta e^{-\frac{\phi a}{\delta} \rho}+a \phi+\alpha_{v}\right) \frac{K \lambda_{A}(\mu)}{b \phi V^{*}} \leq \phi a-\left(\delta+\alpha_{v}\right) \frac{K \lambda_{A}(\mu)}{b \phi V^{*}} .
$$

Thus according to the previous computations, we can deduce that

$$
\mathbf{F}(\rho v(\mu)) \leq \rho\left(\begin{array}{ll}
0 & \frac{b \phi V^{*}}{K} \\
\phi a & -\left(\alpha_{v}+\delta\right)
\end{array}\right) v(\mu)=\rho=\rho \mathbf{F}^{\prime}(\mathbf{0}) v(\mu)
$$

for all $\rho>0$ and $\mu \in(0, \bar{\mu}]$. In addition, using again (30), we show that

$$
\mathbf{F}^{\prime}(\mathbf{0}) \mathbf{1}=\left(\begin{array}{c}
\frac{b \phi V^{*}}{K} \\
\phi a-\left(\alpha_{v}+\delta\right)
\end{array}\right)=\left(\begin{array}{c}
\frac{b \phi V^{*}}{K} \\
\phi a-\delta\left(1-e^{-\frac{\phi a}{\delta}}\right)-\left(\alpha_{v}+\delta e^{-\frac{\phi a}{\delta}}\right)
\end{array}\right) \geq \mathbf{F}(\mathbf{1}),
$$

Thus $(\mathrm{K})_{5}$ is verified.

Following [13, Theorem 3.1], we can apply Theorem 15, page 27, and deduce the existence of a wavefront $U(x-c t)$ for each $c \geq \bar{c}$ connecting $\mathbf{0}$ and $\mathbf{1}$.

\subsection{Existence of traveling-wave solutions for system (18)}

Setting $i_{p}=\frac{I_{p}}{K}, i_{v}=\frac{I_{v}}{V^{*}}$, and $v=\frac{V}{V^{*}}$, system (18) leads to the following

$$
\left\{\begin{array}{l}
\frac{\partial i_{p}}{\partial t}=\frac{b \phi V^{*}}{K}\left(1-i_{p}\right) i_{v} \\
\frac{\partial i_{v}}{\partial t}=D \frac{\partial^{2} i_{v}}{\partial x^{2}}+\phi a\left(v-i_{v}\right) i_{p}-\left(\delta e^{-\frac{\phi a i_{p}}{\delta}}+\alpha_{v}\right) i_{v} \\
\frac{\partial v}{\partial t}=D \frac{\partial^{2} v}{\partial x^{2}}+\left(\alpha_{v}-\mu_{1}\right) v(1-v) .
\end{array}\right.
$$

Setting $u=\left(i_{p}, i_{v}, v\right)$, the previous system can be rewritten as

$$
\left\{\begin{array}{l}
\frac{\partial \mathbf{u}}{\partial t}=\mathbf{D u}+\mathbf{G}(\mathbf{u}), \forall(x, t) \in[0, L] \times(0, T) \\
\mathbf{u}(0, x)=\mathbf{u}_{0}(x)
\end{array}\right.
$$

with

$$
\mathbf{D}=\left(\begin{array}{ccc}
0 & 0 & 0 \\
0 & D & 0 \\
0 & 0 & D
\end{array}\right) \quad \text { and } \quad \mathbf{G}(\mathbf{u})=\left(\begin{array}{c}
\frac{b \phi V^{*}}{K}\left(1-i_{p}\right) i_{v} \\
\phi a\left(v-i_{v}\right) i_{p}-\left(\delta e^{-\frac{\phi a i_{p}}{\delta}}+\alpha_{v}\right) i_{v} \\
\left(\alpha_{v}-\mu_{1}\right) v(1-v)
\end{array}\right)
$$


Obviously, system (32) is a monotone and cooperative system. It is also straightforward to show that the solution $\mathbf{u}$ verifies $\mathbf{0} \leq \mathbf{u} \leq \mathbf{1}$, where $\mathbf{0}=(0,0,0)$ and $\mathbf{1}=\left(1, i_{v}^{\infty}, 1\right)$, where $i_{v}^{\infty}$ is defined in (25), page 14 . It is also important to notice that system (32) admits four equilibria $\mathbf{0}, \mathbf{0}_{v}=(0,0,1), \mathbf{0}_{K}=(1,0,0)$, and 1. The first three are unstable, while the last one is stable. The system being monotone, traveling-wave solutions between $\mathbf{0}$ and $\mathbf{0}_{v}$ or $\mathbf{0}_{K}$ cannot exist. Let us turn to the case $\left[w_{-}, w_{+}\right]$where $w_{-}=\mathbf{1}$, and $w_{+}=\mathbf{0}$ or $w_{+}=\mathbf{0}_{v}$.

Cases where $w_{+}=\mathbf{0}_{v}$ or $w_{+}=\mathbf{0}_{K}$ are related to the sub-cases, studied in sections 3.2 and 3.1. Thus, we know that for these cases, traveling-wave solutions exist and we were able to provide some informations about the traveling-wave speed.

In fact, only the last case remains: $w_{+}=\mathbf{0}$ and $w_{-}=\mathbf{1}$. Unfortunately, the two results used in sections 3.1 and 3.2 do not apply here: first because the system is partially degenerate, and second because a straightforward computations show that $\mathbf{G}^{\prime}(\mathbf{0})$ is not irreducible, but in fact is in Froebenius form

$$
\mathbf{G}^{\prime}(\mathbf{0})=\left(\begin{array}{ccc}
0 & \frac{b \phi V^{*}}{K} & 0 \\
0 & -\left(\alpha_{v}+\delta\right) & 0 \\
0 & 0 & \left(\alpha_{v}-\mu_{1}\right)
\end{array}\right) .
$$

Another possibility is to verify Hypotheses 2.1 (see page 27) and apply Theorem 4.2 of [27]. Unfortunately the positive eigenvalue of $\mathbf{G}^{\prime}(\mathbf{0})$, i.e., $\alpha_{v}-\mu_{1}$, has an eigenvector with only one positive component.

Finally, the last possibility is to consider a small parameter $\varepsilon>0$ such that system (32) is replaced by

$$
\left\{\begin{array}{l}
\frac{\partial \mathbf{u}_{\varepsilon}}{\partial t}=\mathbf{D}_{\varepsilon} \mathbf{u}_{\varepsilon}+\mathbf{G}\left(\mathbf{u}_{\varepsilon}\right), \forall(x, t) \in[0, L] \times(0, T), \\
\mathbf{u}_{\varepsilon}(0, x)=\mathbf{u}_{0}(x),
\end{array}\right.
$$

with $\mathbf{D}_{\varepsilon}=\operatorname{diag}\left(\varepsilon^{2}, D, D\right)$. System (33) is a non-degenerate monotone and cooperative system. Thus, we can apply standard results, like [40, Theorem 3.2, page 178], to show the existence of a Mono-stable traveling wave between $\mathbf{0}$ and $\mathbf{1}$, and then, let $\varepsilon \rightarrow 0$. However, even, with this regularization, we are not able to get information about the traveling waves. The numerical simulations in the next section show that the infected compartments may travel at a lower speed than the vector population. Since we consider additional compartments, $L_{p}$ and $R_{p}$, the infected compartments will exhibit pulse traveling solution, that vanish at both endpoints.

\section{Simulations}

In this section we present numerical simulations to illustrate the previous results and we go further to discuss prevention and control strategies. In particular, we emphasize the impact of the virus lifespan, $1 / \delta$, and the contact rate, $\phi$. Since, we consider a one-dimensional PDE system, we use the well known method of lines: first discretizing in space, and then in time. We consider the finite difference method for the spatial discretization, and the nonstandard finite difference method for the time discretization (see for instance [2] and references therein).

The parameters values used in the forthcoming simulations are summarized in Table 2, page 19 .

\begin{tabular}{|l||c|c|c|c|c|c|c|c|c|c|c|c|}
\hline Parameter & $D$ & $K$ & $a$ & $b$ & $k_{1}$ & $k_{2}$ & $\alpha_{v}$ & $\beta$ & $\delta$ & $\mu_{1}$ & $\mu_{2}$ & $V^{*}$ \\
\hline Value & 1 & 100 & 0.2 & 0.2 & 0.8 & 0.5 & 0.4 & 0.01 & 0.2 & 0.1 & 0.001 & 300 \\
\hline
\end{tabular}

Table 2: Parameters values

In Figs. 7, we present simulations related to system (21), page 14, when the whole crop is already infected, i.e. $I_{p}=K$. Thus, no surprise: as expected from the theory, the infected vectors spread like the total vector-population, at speed $c_{v}$, whatever the values taken for $\delta$ and $\phi$. According to (19), $I_{v}^{\infty}$ depends on $\delta$ and $\phi$. Thus, as expected, the larger (lower) the values of $\delta(\phi)$, the lower $I_{v}^{\infty}$.

Next, we consider the full subsystem (18), page 13 in two cases: when the vector population is not established and when it is already established, i.e. $V=V^{*}$, or equivalent (26), page 15 . One infected vector 

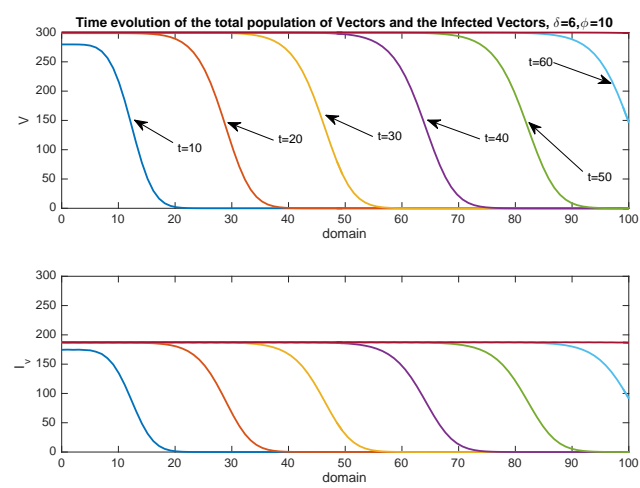

(a) $\delta=6$ and $\phi=10$.


(c) $\delta=6$ and $\phi=5$.
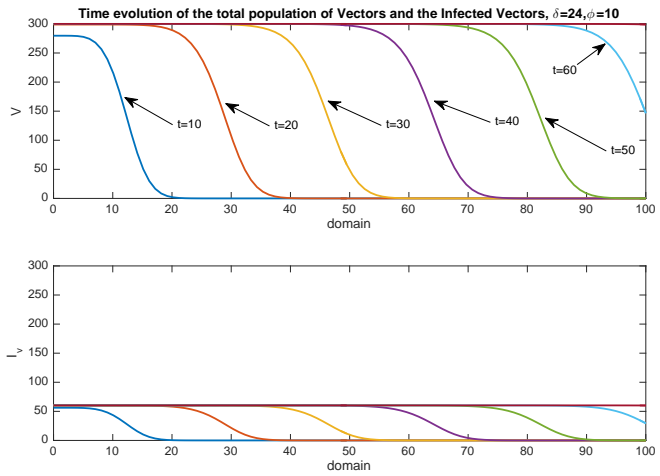

(b) $\delta=24$ and $\phi=10$.
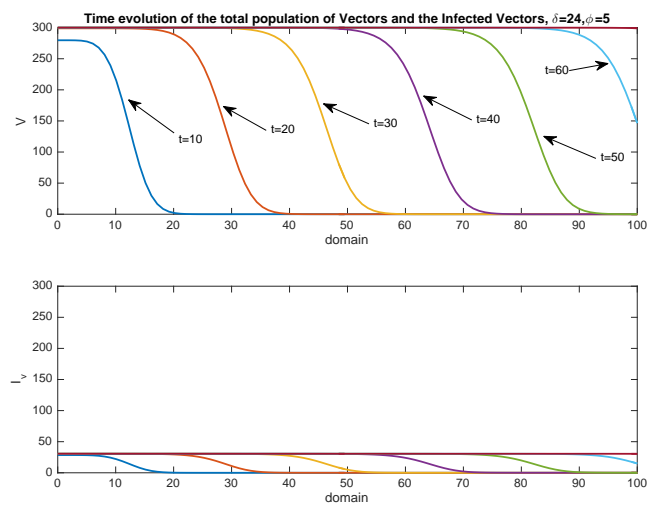

(d) $\delta=24$ and $\phi=5$.

Figure 7: Simulations of subsystem (21) when the crop is still infected, with different values for the virus lifespan, $\delta$, and the contact rate, $\phi$.

is introduced at $x=0$. In Fig. 8, page 20, we represent the estimated velocity of the infected front versus $\delta$, the virus decay rate for two main cases: Fig. 8(a) when the vector population $V$ is not established and Fig. 8(b) when it is still established, $V=V^{*}$. As expected, the larger $\delta$, the lower the speed of the infected front, while the velocity of the pest population is constant. The contact rate $\phi$ also impacts the velocity of the infected front: compare the two curves.

When the vector population is still established, it is interesting to notice that, for $\delta$ small enough, the infected pest front can travel faster than the pest front when invading the crop (compare with the line in Fig. 8(a)). In fact, as shown in Fig. 8(a), the pest front velocity acts as an upper bound for the infected fronts, while it is not the case in Fig. 8(b). This particular result may have a biological explanation through the Vector Manipulation Hypothesis discussed earlier. Indeed, when $\delta$ is small and less than unit, it means that the virus is semi-persistent or persistent and thus circulative, meaning that the virus replicates inside the vectors. It is well documented in the literature that virus may change the behavior of the insect thereby facilitating their spreading ability looking for susceptible hosts (plants). Also, when $\delta$ is large, it means that the lifespan of the virus is short (non-persistent) and thus cannot have an impact on the behavior of the insect. That is why the curves in Figs. 8(a) and 8(b) lead to the same velocity for a given $\delta$, whether the vector population is established or not.

Finally, we present some simulations of the full system (9)-(10), page 10. We consider also two cases: $\gamma=0$ and $\gamma=0.2$, i.e., with and without sanitary harvest. In Figs. 9 and 10, we show the impact of $\delta$ on the speed of the Pest-Infective-front. We also show that $\phi$ may have a big impact on the velocity (compare Figs. 9 and 10). Indeed, reducing $\phi$ slows down rapidly the spread of the infection, when $\delta>\delta^{*}$, where 


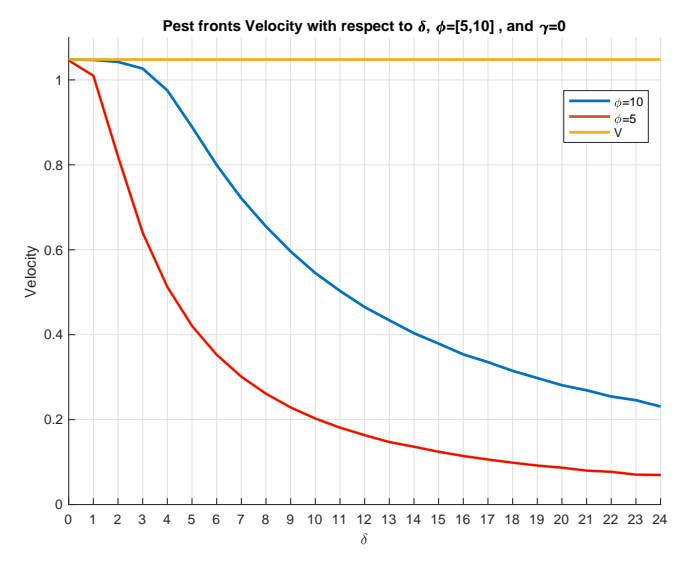

(a) Pest population is not established.

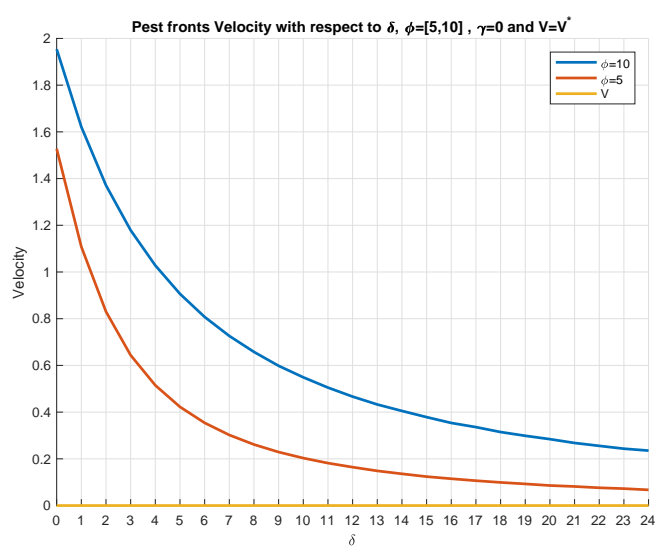

(b) Pest population is established.

Figure 8: Simulations of subsystem (18): Traveling-wave velocity versus $\delta$, with or without established population

$\delta^{*}=15(7)$ when $\gamma=0(0.2)$. In particular as $\delta$ increases, the front speed decays to zero, more or less rapidly according to $\phi$. Finally, it is interesting to notice that the harvest rate has an impact only when $\phi$ is small: compare Fig. 10(a) and Fig. 10(b), page 21.

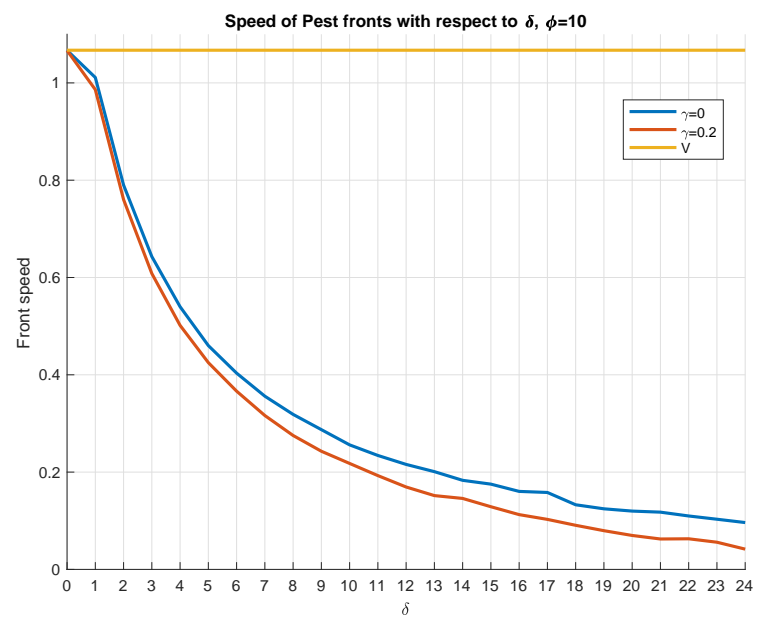

Figure 9: Simulations of Model (9)-(10). Speed of the pest front and the infected pulse wave when $\phi=10$, with $(\gamma=0.2)$ and without harvest $(\gamma=0)$

In Fig. 11, when $\gamma=0$, then all plants become resistant. Like in the previous sub-models, the pulse traveling waves related to the infected compartments have a lower speed than the total vector population. However, the wave speed is even lower because we have several additional compartments, $L_{p}$ and $R_{p}$, that introduce an additional delay.

Altogether, qualitatively, for the full model (9)-(10), we obtain a behavior almost similar to the submodels: the speed of the infective pulse wave reduces (strongly) according to the virus lifespan and the contact rate with the crops. 


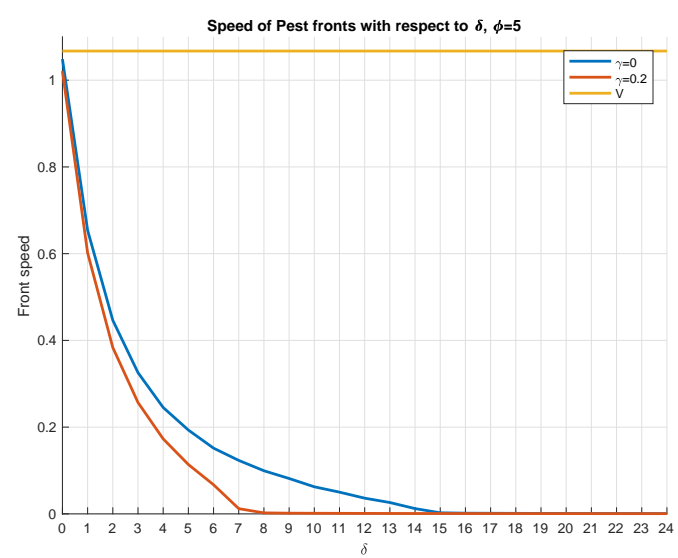

(a) Overall view.

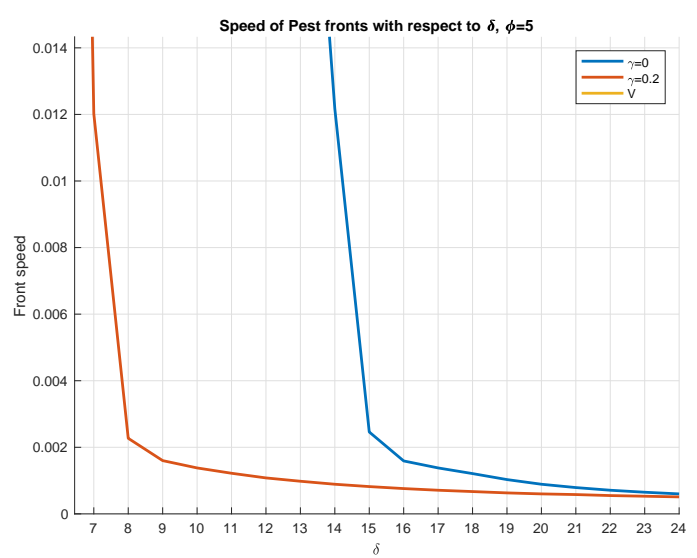

(b) Zoom on interval $[7,24]$.

Figure 10: Simulations of model (9) - (10). Speed of the pest front wave and the infected pulse wave when $\phi=5$, with $(\gamma=0.2)$, and without harvest $(\gamma=0)$.
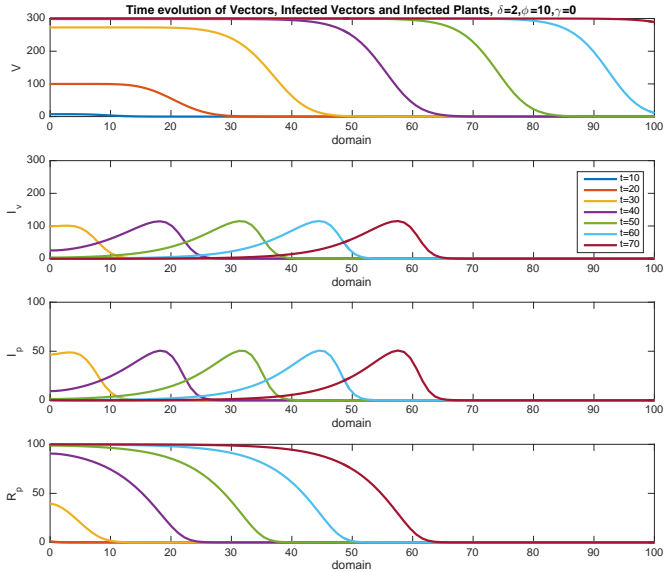

(a) $\delta=2, \phi=10$, and $\gamma=0$.
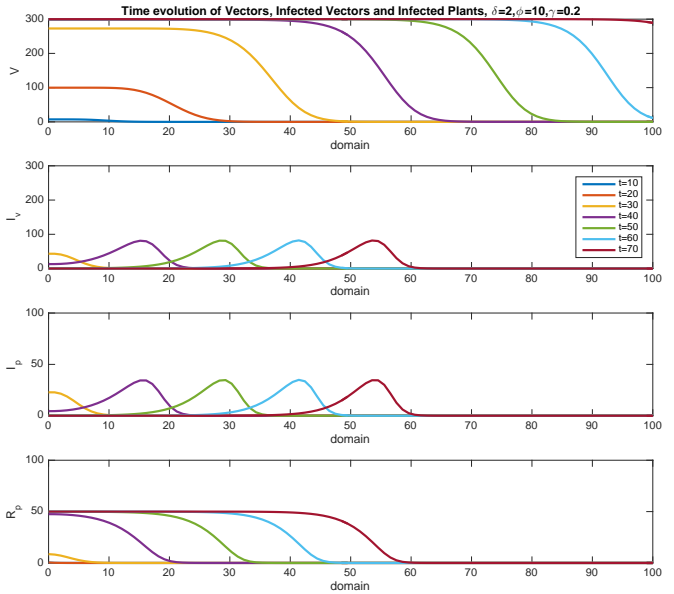

(b) $\delta=2, \phi=10$, and $\gamma=0.2$.

Figure 11: Simulations of system (9) - (10) without and with sanitary harvest

\subsection{Prevention and Control}

In terms of prevention, it is important to capture the right invasive front, i.e., the infective front. Our results show that the lower the lifespan of the virus, the lower the speed of the infective front, meaning that diseases may appear in the field far after the initial invasion of (healthy) pest.

In general, people in charge of pest control or prevention usually recommends setting-up of surveillance programs (using traps) during critical times of plant growth in order to start the control immediately after the arrival of the first pests (or when certain threshold has been reached). Here, clearly, according to our results, this strategy is not possible. In addition to the surveillance protocol, virus-detection using a real time PCR analysis ${ }^{1}$, would be a better strategy in order to capture the right front(s), i.e., the infected front(s),

\footnotetext{
${ }^{1}$ real time PCR (polymerase chain reaction) is a biological technique for the detection and expression analysis of gene(s) in real-time; see the review and applications of real-time PCR [8] on some plants $[38,18]$
} 
and thus start control strategies at the right time.

In terms of control, let us consider the same approach as in the temporal model where barrier plants are used as inter-crops. This leads again to a change in the contact rate. For instance, if we assume that the percentage of barrier plants over the "cash" crop is 16,67\%, then, we show in Fig. 12, page 23, that we velocity of the infected pulse wave slows down, compared to Fig. 9, page 21. When $\delta=0$, it is also interesting to notice that barrier plants have absolutely no effect.

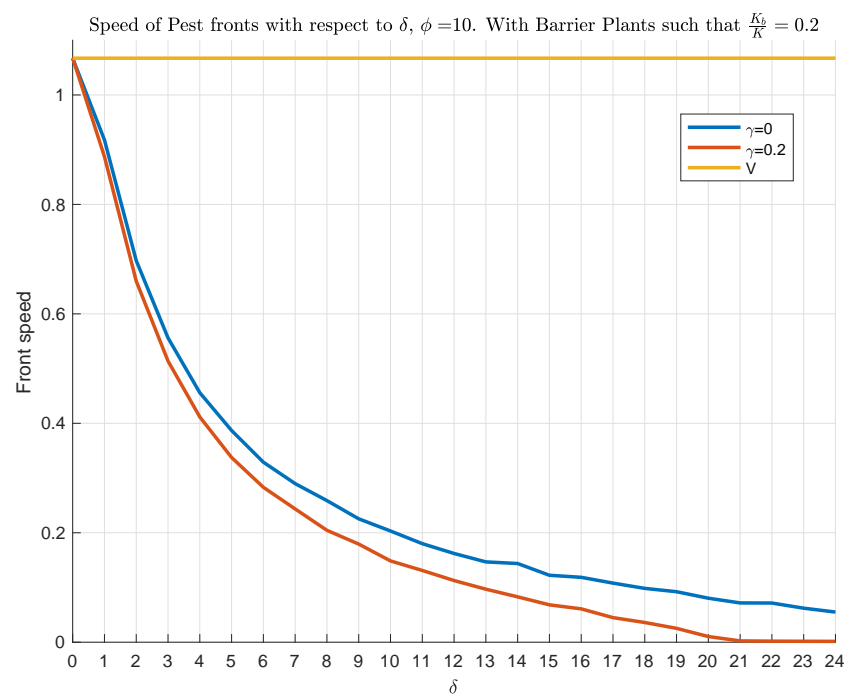

Figure 12: Simulations of system (9)-(10) with inter-cropping Barrier Plants, with and without sanitary harvest

Thus the concept of plant barriers can be very effective to reduce the invasion of infected pest. However, as it is modeled, we don't take into account various possibility, and in particular, the fact that barrier plants can be used, not as inter-crop, but also as a barrier that may surround the cash crop. For instance, maize is often used as a barrier crop along the borders of tomato field, thus preventing the entry of whitefly (see Fig. 13, page 23, obtained using Simeo simulator implemented in the AMAPstudio framework [20]).

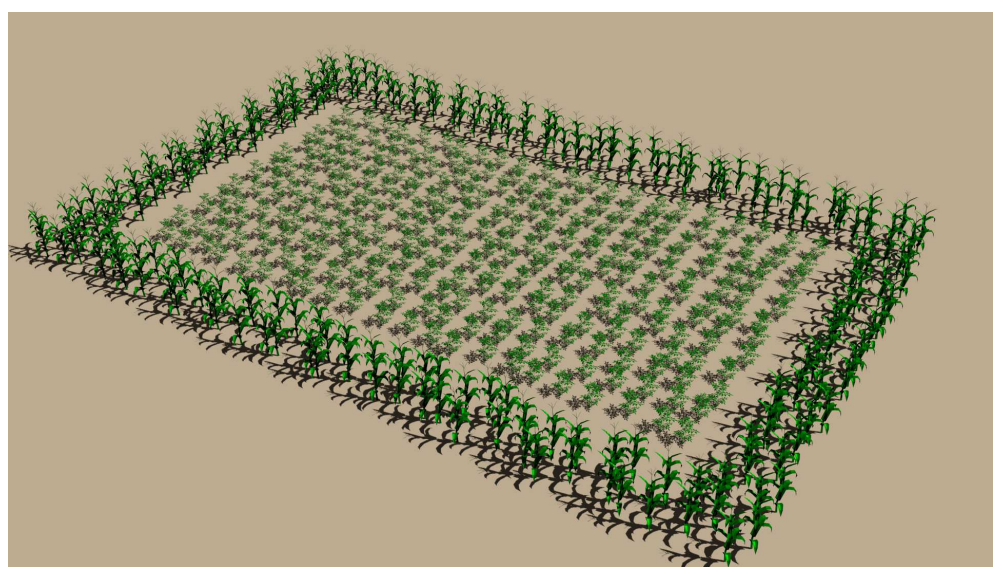

Figure 13: Maize as Barrier plants to protect Tomato plants 


\section{Conclusions}

Crop vector-borne disease modeling can lead to new and exciting mathematical problems, for which the existing theory is not always applicable. However, using suitable sub-models, and in particular models that enter the theory of monotone dynamical systems [36], we are able to get some insights into the resulting partially degenerate non-monotone systems.

Here, in the case of non-persistent virus, we showed that the virus lifespan, $1 / \delta$, can have an important impact on the speed of the infected traveling front, such that the infected vectors can invade a long time after the whole pest population has invaded the crop. The contact rate, $\phi$, may also have the same impact on the infective front. Altogether, this may have strong implications on the surveillance and/or the control strategies.

It is now well acknowledge that viruses (parasites and pathogens, in general) can induce changes in host or/and vector behavior to enhance their transmission [22], such that the dynamics of the whole system can become more complex. Of course for semi-persistent viruses, recent observations seem to indicate that the Vector Manipulation hypothesis [22] can impact the spreading of aphids in the field, preferably to susceptible plants, which in some sense may speed-up the infective front as seen in Fig. 8, page 20. All these facts show how complex and diverse pest-virus-crop systems are. In other words, our model, though generic, can be improved or adapted in order to take into account specific facts related to a particular diseases.

In terms of control strategies or crop protection, we focused mainly on barrier plants or crops, because it is a reliable and sustainable strategy. Indeed, it is based on an accurate knowledge on pest biology and ecology and its interaction with the host plant(s). In this paper we started with a very simple use of barrier plants, showing that it can be efficient. However, we need to consider more realistic models in order to take into account concrete use of barrier plants. This implies considering more complex spatio-temporal models.

Of course other control strategies could be taken into account for comparison or in combination. For instance, eco-friendly nets [37] may be a good and sustainable control strategy, to reduce $\phi$. However, the use of nets may change locally the environmental parameters (temperature, humidity) of the plants, and thus may have an impact on their (photosynthetic) growth, and eventually, foster other kind of diseases, e.g., fungi. In fact, there exists several other control strategies, including the use of natural enemies, natural pesticides or pesticides extracted from plants, fungi like Beauveria bassiana, etc, and traps. Indeed, traps are widely used around the world, using pheromones or food to attract and catch insects [4], and/or for mating disruption [5].

From the plant perspective, additional complexity could be taken into account if we add plant growth dynamics, since we know that plant's attractiveness may change according to physiological stages.

From the Mathematical perspective, the previous models need further investigations in order to get an estimate or bounds for the infected pulse-wave or front-wave velocity. Two-dimensional extension of our PDE-ODE model is also necessary in order to be even more realistic. And, finally, comparison with real experiments in order to validate/modify/adapt our models is mandatory.

Acknowledgments: YD would like to thank the PHC PROTEA program $n^{\circ} 33879$ that supported partly this work. MC acknowledges the support of the South African Research Chairs Initiatives (SARChI Chair) in Mathematical Models and Methods in Bioengineering and Biosciences. Thanks are also addressed to the anonymous reviewers whose suggestions have contributed to the improvement of the paper.

\section{References}

[1] Anderson R.M., May R., 1991. Infectious Diseases of Humans: Dynamics and Control, Oxford University Press, Oxford, UK.

[2] Anguelov R., Dumont Y., Lubuma J. M.-S., 2012. On nonstandard finite difference schemes in biosciences, AIP Conference Proceedings 1487 (1), 212223.

[3] Anguelov R., Lubuma J.M.-S. , Dumont Y., 2012. Mathematical analysis of vector-borne diseases on plants. In Guo, Y., Kang, M. Z., Dumont, Y. (Eds) Plant growth modeling, simulation, visualization and 
applications. Proceedings PMA12 : The Fourth International Symposium on Plant Growth Modeling, Simulation, Visualization and Applications, Shanghai, China, 31 October-3 November 2012. Beijing: IEEE Press, 22-29 p.

[4] Anguelov, R., Dufourd, C., Dumont, Y., 2017. Simulations and parameter estimation of a trap-insect model using a finite element approach. Mathematics and Computers in Simulation, 133 : 47-75.

[5] Anguelov, R., Dufourd, C., Dumont, Y., Mathematical model for pest-insect control using mating disruption and trapping. eprint arXiv:1608.04880. Under review.

[6] Blanc, S.; Michalakis, Y., 2016. Manipulation of hosts and vectors by plant viruses and impact of the environment, Curr. Opin. Insect Sci. (16), 18

[7] Brault V., Uzest M., Monsion B., Jacquot E., Blanc S., 2010. Aphids as transport devices for plant viruses. C. R. Biol. 333 (6-7), 524-538.

[8] Deepak, S., Kottapalli, K., Rakwal, R., Oros, G., Rangappa, K., Iwahashi, H., Agrawal, G., 2007. Real-Time PCR: Revolutionizing Detection and Expression Analysis of Genes. Current Genomics 8(4), 234-251.

[9] Diekmann O., Heesterbeek J.A.P., and Metz J.A.J., 1990. On the definition and the computation of the basic reproduction ratio R0 in models for infectious diseases in heterogeneous populations, J. Math. Biol.,28, 365-382.

[10] Döring T.F, Röhrig K., (2016). Behavioural response of winged aphids to visual contrasts in the field. Ann Appl Biol, 168, 421-434. doi:10.1111/aab.12273

[11] Dumont Y., Chiroleu F., Domerg C., 2008. On a temporal model for the Chikungunya disease: modeling, theory and numerics, Math. Biosc. 213, 70-81.

[12] Dumont Y., Chiroleu F., 2010. Vector control for the chikungunya disease, Math. Biosc. Eng. 7, 313345.

[13] Fang J., Zhao X.Q., 2009. Monotone wavefronts for partially degenerate reaction-diffusion systems, J. Dynam. Differential Equations, 21, 663-680.

[14] Fereres A., Raccah B., 2015. Plant Virus Transmission by Insects. Encyclopedia of Life Sciences (ELS). John Wiley \& Sons.

[15] Fereres A., Peaflor M.F.G.V., Favaro C.F., Azevedo K.E., Landi C.H., Maluta N.K., Bento J.M., Lopes J.R., 2016. Tomato infection by whitefly-transmitted circulative and non-circulative viruses induce contrasting changes in plant volatiles and vector behaviour. Viruses, 8, 225, 15 p.

[16] Fife P.C., 1979. Mathematical Aspect of Reacting and Diffusing Systems, volume 28 of Lecture Notes in Biomathematics. Springer, Berlin.

[17] Fitzgibbon, W.E., Langlais, M., Morgan, J.J.: A mathematical model for indirectly transmitted diseases. Math Biosci., 206 , 2007, 233-248.

[18] Froissart, R., Doumayrou, J., Vuillaume, F., Alizon, S., Michalakis, Y., 2010. The virulencetransmission trade-off in vector-borne plant viruses: a review of (non-)existing studies. Philosophical Transactions of the Royal Society B: Biological Sciences, 365(1548), 19071918.

[19] Gray S. M., Banerjee N., 1999. Mechanisms of Arthropod Transmission of Plant and Animal Viruses, Microbiol Mol Biol Rev. 63(1), 128-148.

[20] Griffon S., de Coligny F., 2014. AMAPstudio: an Editing and Simulation Software Suite for Plants Architecture Modelling. Ecological Modelling 290, 3-10.

[21] Hollis S.L., Morgan J.J., 1992. Partly dissipative reaction-diffusion systems, Nonlinear Analysis, TMA, vol. 19 (5), 427-440. 
[22] Ingwell L.L., Eigenbrode S.D., Bosque-Prez N.A., 2012. Plant viruses alter insect behavior to enhance their spread. Sci Rep, 2, p. 578

[23] Jeger M.F., van den Bosch F., Madden L.V., Holt J., 1998. A model for analysing plant-virus transmission characteristics and epidemic development, IMA J. Math applied in Med and Biol 15, 1-18.

[24] Jones D.R., 2003. Plant viruses transmitted by whiteflies. Eur. J. Plant. Pathol. 109, 195-219.

[25] Lewis, M.A., Li, B., Weinberger, H.F. (2002) Spreading speed and the linear determinacy for two-species competition models. Journal of Mathematical Biology, 45(3):219-233.

[26] Li B., Weinberger H. F., Lewis M. A., 2005. Spreading speeds as slowest wave speeds for cooperative systems, Mathematical Biosciences, 196, 82-98.

[27] Li B., 2012. Traveling wave solutions in partially degenerate cooperative reaction-diffusion systems, Journal of Differential Equations 252, 4842-4861.

[28] Macdonald G., 1957. The Epidemiology and Control of Malaria, Oxford University Press, London.

[29] Marion M., 1989. Finite-Dimensional Attractors Associated with Partly Dissipative Reaction-Diffusion Systems, SIAM Journal on Mathematical Analysis 20(4), 816-844.

[30] Nault L.R., 1997. Arthropod transmission of plant viruses: a new synthesis, Ann. Entomol. Soc. Am. 90, 521-541.

[31] Navas-Castillon J., Fiallo-Olivé E., Snchez-Campos S., 2011. Emerging virus diseases transmitted by whiteflies. Annu. Rev. Phytopathol. 49, 219248.

[32] Palacios I., Drucker M., Blanc S., Leite S., Moreno A., Fereres A., 2002. Cauliflower mosaic virus is preferentially acquired from the phloem by its aphid vectors. J Gen Virol. 83, 3163-3171.

[33] Rauch, J., Smoller, J., 1978. Qualitative theory of the FitzHugh-Nagumo equations. Advances in Mathematics, 27(1), 12-44

[34] Ross R., 1911. The Prevention of Malaria, John Murray, London.

[35] Rothe F., 1984. Global solutions of reaction-diffusion systems. Lecture notes in mathematics, vol. 1072. Springer-verlag, Berlin(West)-Heidelberg-New York-Tokyo.

[36] Smith H., 2008. Monotone dynamical systems: an introduction to the theory of competitive and cooperative systems, Amer. Math. Soc. 41.

[37] Mutisya S., Saidi M., Opiyo A., Ngouajio M., Martin T., 2016. Synergistic effects of agronet covers and companion cropping on reducing whitefly infestation and improving yield of open field-grown tomatoes. Agronomy (Basel), 6 (42), 14 p.

[38] Thomson D., Dietzgen R.G., 1995. Detection of DNA and RNA plant viruses by PCR and RT-PCR using a rapid virus release protocol without tissue homogenization, J. of Virological Methods 54 (2), $85-95$.

[39] Van den Driessche P., Watmough J., 2002. Reproduction numbers and sub-threshold endemic equilibria for compartmental models of disease transmission, Math. Biosciences 180, 29-48.

[40] Volpert A., Volpert Vl., Volpert V., 1994. Traveling wave solutions of parabolic systems. Translation of Mathematical Monographs, Vol. 140, Amer. Math. Society, Providence.

[41] Weinberger H.F., Lewis M.A., Li B., 2002. Analysis of linear determinacy for spread in cooperative models. J Math Biol. 45(3):183-218. 


\section{Annexe}

\subsection{Existence result for Monostable wavefronts}

Here we recall some results proved in [13]. We consider the following system of $n(\geq 2)$ equations

$$
\frac{\partial u_{i}}{\partial t}=d_{i} \Delta u_{i}+f_{i}\left(u_{1}, \ldots, u_{n}\right), \quad t \geq 0, \quad x \in \mathbb{R}, \quad 1 \leq i \leq n,
$$

with $f: \mathbb{R}^{n} \rightarrow \mathbb{R}^{n}$ a continuous function. Let $D=\operatorname{diag}\left(d_{1}, \ldots, d_{n}\right), A(\mu)=\mu^{2} D+f^{\prime}(\mathbf{0})$, and $\lambda_{A}(\mu)$ the stability modulus of $A(\mu)$. Since $f^{\prime}(\mathbf{0})$ is cooperative and irreducible, then $\lambda(u)>0$, whatever $\mu>0$, such that we can define $\Phi(\mu)=\frac{\lambda(\mu)}{\mu}$. Then from [13, Lemma 2.1], $\bar{c}=\min _{\mu>0} \Phi(\mu) \geq 0$ is defined.

Assume that $f$ satisfy the following conditions $(\mathrm{K})$ :

1. $f$ is continuous with $f(\mathbf{0})=f(\mathbf{1})=\mathbf{0}$, and there is no $\eta$ other than $\mathbf{0}$ and $\mathbf{1}$ such that $f(\eta)=\mathbf{0}$ and $\mathbf{0} \leq \eta \leq \mathbf{1}$.

2. System (34) is cooperative.

3. $f(u)$ is piecewise continuously differentiable in $u$ for $\mathbf{0} \leq u \leq \mathbf{1}$ and differentiable at $\mathbf{0}$, and the matrix $f^{\prime}(\mathbf{0})$ is irreducible with $s\left(f^{\prime}(\mathbf{0})\right)>0$.

4. There exists $a>0, \sigma>1$ and $r>0$ such that $f(u) \geq f^{\prime}(\mathbf{0}) u-a\|u\|^{\sigma} \mathbf{1}$ for all $\mathbf{0} \leq u \leq \mathbf{r}$.

5. For any $\rho>0, f(\min \{\rho v(\mu), \mathbf{1}\}) \leq \rho f^{\prime}(0) v(\mu), \forall \mu \in\left(0, \mu^{*}\right]$, where $\mu^{*}$ is the value of $\mu$ at which $\Phi(\mu)$ attains its infimum.

We have the following results

Lemma 14 (Lemma $2.3[13])$. Assume that assumptions $(K)_{1,2,3}$ hold. Let $\phi \in C_{1}$ and $u(t, x: \phi)$ be the unique solution of (the integral form) (34). Then, there exists a real number $c^{*} \geq \bar{c}>0$ such that the following statements are valid

- If $\phi$ has compact support, then $\lim _{t \rightarrow \infty,|x| \geq c t} u(t, x: \phi)=\mathbf{0}, \forall c>c^{*}$.

- For any $c \in\left(0, c^{*}\right)$ and $r>0$, there is a positive number $R_{r}$ such that for any $\phi \in C_{1}$ with $\phi \geq r$ on an interval of length $2 R_{r}$, there holds $\lim _{t \rightarrow \infty,|x| \leq c t} u(t, x: \phi)=\mathbf{1}$

- If, in addition, $f\left(\min \left\{\rho v\left(\mu^{*}\right), \mathbf{1}\right\}\right) \leq \rho f^{\prime}(\mathbf{0}) v\left(\mu^{*}\right), \forall \rho>0$, then $c^{*}=\bar{c}$.

Theorem 15 (Theorem 3.1 [13]). Assume (K) holds, and let $c^{*}$ be defined as in Lemma (14). Then for each $c \geq c^{*}$, system (34) has a nondecreasing wavefront $U(x+c t)$ connecting $\mathbf{0}$ and $\mathbf{1}$; while for any $c \in\left(0, c^{*}\right)$, there is no wavefront $U(x+c t)$ connecting $\mathbf{0}$ and $\mathbf{1}$.

Assuming now that $f$ verifies the following

\section{Hypothesis 2.1 in [27] :}

1. There is a proper subset $\Sigma_{0}$ of $\{1, \ldots, k\}$ such that $d_{i}=0$ for $i \in \Sigma_{0}$ and $d_{i}>0$ for $i \Sigma_{0}$.

2. $f(\mathbf{0})=\mathbf{0}$, there is a constant $\beta>>0$ such that $f(\beta)=\mathbf{0}$ which is minimal in the sense that there are no constant $\nu$ other than $\beta$ such that $f(\nu)=0$ and $\mathbf{0}<<\nu<<\beta$, and the equation $f(\alpha)=0$ has a finite number of constant roots.

3. The system is cooperative;

4. $f(\alpha)$ is uniformly Lipschitz continuous in $\alpha$ so that there is $\rho>0$ such that for any $\alpha_{i} \geq 0, i=1,2$, $\left|f\left(\alpha_{1}\right)-f\left(\alpha_{2}\right)\right| \leq \rho\left|\alpha_{1}-\alpha_{2}\right|$.

5. $f$ has the Jacobian $f^{\prime}(\mathbf{0})$ at $\mathbf{0}$ with the property that $\left.f^{\prime} \mathbf{0}\right)$ has a positive eigenvalue whose eigenvectors has positive components

Then, according to Theorem 4.2 [27], we have existence of a traveling wave solution, connecting $\mathbf{0}$ to $\beta$. 